



OUVRAGE PUBLIE SOUS LES AUSPICES DU MINISTERE DE L'TNSTRUCTION PUBLIQUE SOUS LA DIRECTION DE L. JOUBIN PRORESSEUR AU MUSEUM D'HISTOIRE NATURELLE

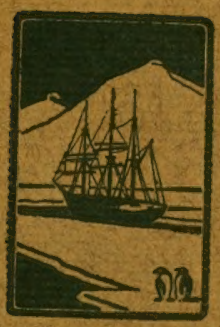

\title{
DEUXIEME EXPEDITION
}

\section{ANTARCTIQUE FRANÇAISE}

\author{
$(1908-1910)$ \\ COMMANDEE PAR LE \\ $D^{r}$ JEAN CHARCOT
}

SCIENCES NATURELLES : DOCUMENTS SCIENTIFIQUES

GASTROPODES PROSOBRANCHES, SCAPHOPODE

ET PELECCYPODES

PAR ED. LAMY

AMPHINEURES

PAR JOH. THIELE

MASSON ET CIE. ÉDITEURS

120. Bd SAINT.GERMAIN, PARIS (VI\%)

1911 


\section{Commission Chargée par l'A CAdémie des SCIENCES d'élaborer le programme scientifique de l'Expédition}

MM. les Membres de I'Institut :

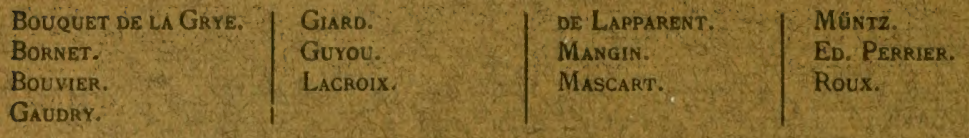

Commission nommé par le Ministère de l'Instruction Publique pour examiner les résultats scientifiques de l'Expédition

MM. Ed. Perrier......... Membre de I'Institut, Directeur du Muséum d'Histoire naturelle, Président.

Vice-Amiral Fournier, Membre du Bureau des Longitudes, Vice-Président.

Angor ............. Directeur du Bureau central météorologique.

BAYET ............ Correspondant de l'Institut, Directeur de l'Enseignement supérieur.

Bigourdan .......... Membre de l'Institut, Astronome a l Observatoire de Paris.

Colonel Bouraeols. .. Directeur du Service géographique de l'Armée.

Bouvier........... Membre de I'Institut, Professeur au Muséum d'Histoire naturelle.

GrAviER........... Assistant au Muséum d'Histoire naturelle.

Commandant Guyou.. Membre de I'Institut, Membre du Bureau des Longitudes.

HanussE ............ Directeur du Service hydrographique au Ministère de la Marine.

Joubin............. Professeur au Muséum d'Histoire naturelle et a l'Institut Oçanographique.

LACrOIX ............ Membre de l'Institut, Professeur au Muséum d'Histoire naturelle.

Lallemand......... Membre de IInstitut, Membre du Bureau des Longitudes, Inspecteur général des mines.

Lippmann .......... Membre de l'Institut, Professeur à la Faculté des Sciences de l'Université de Paris.

Müntz ............. Membre de l'Institut, Professeur a l'Institut agronomique.

Rabot............. Membre de la Commission des Voyages et Missions scientifiques et littéraires.

Roux .............. Membre de l'Institut, Directeur del'Institut Pasteur.

VéLAiN ............ Professeur à la Faculté des Sciences de l'Université de Paris. 


\title{
DEUXIËME EXPÉDITION ANTARCTIQUE FRANÇAISE
}

\author{
$(1908-1910)$ \\ COMMANDEE PAR LE \\ $D^{r}$ JEAN CHARCOT
}

Division of Mollumbers
Sectional Library 


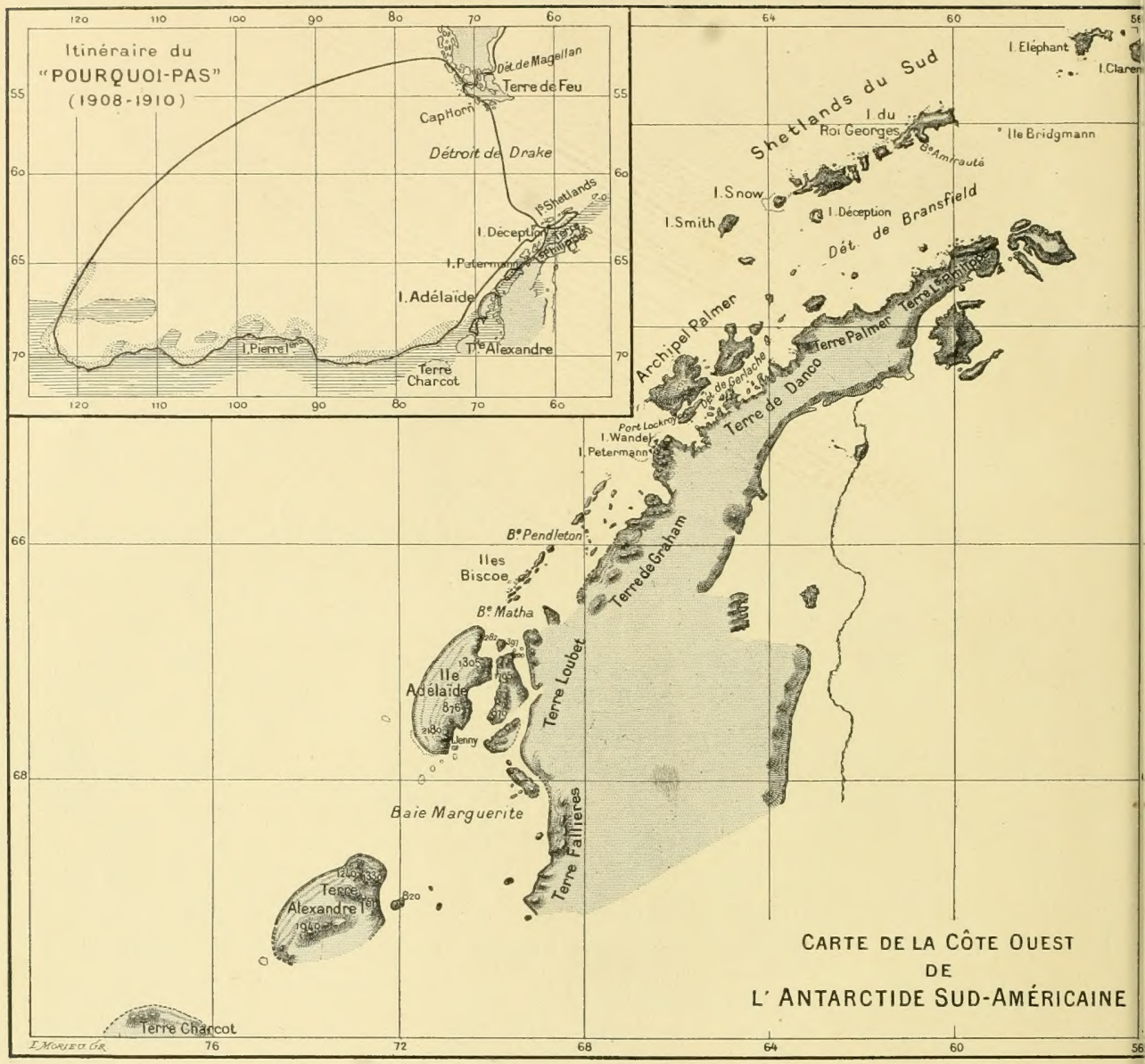

CARTE DES REGIONS PARCOURUES ET RELEVEES PAR L'EXPÉDITION

MEMBRES DE L'ÉTAT MAJOR DU " POURQUOI-PAS "

\section{J.-B. CHARCOT}

M. BONGRAIN

L. GAIN

R.-E. GODFROY

E. GOURDON

J. LiOUVILLE.

J. ROUCH.

A. SENOUQUE.
Hydrographie, Sismographie, Gravitation terrestre, Observations astronomiques.

Zoologie (Spongiaires, Échinodermes, Arthropodes, Oiseaux et leurs parasites) Plankton, Botanique. Marées, Topographie côtière, Chimie de l'air.

Géologie, Glaciologie.

Médecine, Zoologie (Pinnipèdes Cétacés, Poissons, Mollusques, Calenterés, Vermidiens, Vers et Protozoaires, Anatomie comparée, Parasitologie).

Météorologie, Océanographie physique, Electricité atmosphérique.

$\mathrm{M}_{\text {agnétisme terrestre, Actinométrie, Photographie scientifique. }}$ 


\section{DEUXIËME EXPÉDITION}

ANTARCTIQUE FRANÇAISE

$$
(1908-1910)
$$

COMMANDEE PAR LE

Dr JEAN CHARCOT

SCIENCES NATURELLES : DOCUMENTS SCIENTIFIQUES

GASTROPODES PROSOBRANCHES, SCAPHOPODE ET PÉLÉCYPODES

PAR ÉD, LAMY

A M PHINEURES

PAR JOH. THIELE

MASSON ET CIE, ÉDITEURS

$120, B^{d}$ SAINT, GERMAIN. PARIS $\left(V I^{\circ}\right)$

Tous droits de traduction et de reproduction réservés 


\section{LISTE DES COLLABORATEURS}

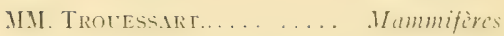

AnTHONY............ Documents embryogéniques.

Liouville .......... Phoques, Cétacés (Anatomie, Biologie).

Grain ............. Oiseaux.

Roule.............. Poissons.

SLUITER ............ Tuniciers.

Joubin............. Céphalopodes, Brachiopodes, Némertiens.

I.AMY............... Gastropodes et Pélécypodes.

VAysSIÈrE ........... Nudibranches.

KeILIN............. Diptères.

Trouessart et Berlfse. Acariens.

Neumaxi.......... Pédiculines, Mallophages, 1xodides.

Bouvier........... Pycnogonides.

Coutière ........... Crustacés Schizopodes et Décapodes.

IIle Richardsox ........... Isopodes.

CaLman............. Cumacés.

DE DADAY............ Entomostracés.

Mil. Chevreux ............. Amphipodes.

CÉPÈDE.............. Copépodes.

QuIDOR............. Copépodes parasites.

Calvet ............. Bryozoaires.

Gravier........... Polychètes, Alcyonaires et Ptérobranches.

HÉRUBEL............ Géphyrients.

Germain et Joubin .... Chétognathes.

RaIlliet et Henry .... Helminthes parasites.

Hallez............. Polyclades et Triclades maricoles.

KenLer ........... Stellérides, Ophiures et Echinides.

VANEY ............. Holothuries.

PAX ................. Actiniaires.

Bill.irn ............ Hydröides.

TOPSENT ........... Spongiaires.

PÉNARD ............. Rhizopodes.

FAURE-FrÉMIET ...... Foraminifères.

Cardot............... Mousses.

Ime LeMorne.............. Algues calcaires.

MiI. Gain............... Algues.

Mangin............ Phytoplancton.

Peragallo.......... Diatomées.

HUE ............... Lichens.

METCHNIKofF ........ Bactériologie.

Gourdon............ Géographie physique, Glaciologie, Pétrographie.

Bongrain........... Hydrographie, Cartes, Chronométrie.

GODFROX ........... Marées.

MIÜNTZ ............. Recherches sur l'atmosphère.

Rouch ............ Météorologie, Océanographic physique.

Senovoue ........... Magnétisme terrestre, Actinométrie.

J.-B. Charcot ......... Journal de l'Expédition. 


\section{GASTROPODES PROSOBRANCHES, SCAPHOPODE ET PÉLÉCYPODES \\ Par ÉDOUARD LAMY \\ ASSISTANT AU MUSECM D'HISTOIRE NATURELLE}

Les Mollusques recueillis par M. le I' Jacques Liouville pendant la deuxième expédition de $\mathrm{II}$. le I) Ciharcot comprennent 19 Gastropodes Prosobranches, 1 Scaphopode ef 2:; Pélécypodes. Sur ces 4 : formes, qui proviennent toutes de l'Antarctique. 2 sont des jeunes spécifiquement indéterminables, 8 sont nouvelles et $3:$; avaient déja étri trouvées lors des différents voyages effectués pendant ces dernières années, comme le montre le tableau ci-joint (p. 3) ; 21 notamment avaient été rapportées par la première expédition du $\mathrm{D}^{\mathrm{r}}$ Charcot (1903-1905).

Il y en a sept observées plus ou moins anciennement dans la région Magellanique : Lavilittorina caliginosa Gld., Nacella fuegiensis Rve,, Limatula pygmxa Phil., Modiolarea trapesina Lk., Limopsis hirtella Mab. et Rochebr., L. Joussenumei Mab. et Rochebr., Ioldia Wooducurdi IIanl.

Dix ont été signalées pour la première fois des îles Kerguelen: Neohuccinum Éaloni Smith, Cominella vestita v. Martens (dont je décris une variété elongatu nouvelle), Eatmmella lierguelonensis Sm., E. raliginnsu Sm., Kellya cardiformis Sm., K. suborbicularis Mtg., Cardita astartoides Irts., Anatina elliptica Kg. et Brod., Thracia meridionalis Sm., Amussium clathratum Mrts.

Deux ont été indiquées de la Géorgie du Sud par v. Martens et Pfeffer : Lacunella antaretica Mrts., Nacella polaris IIombr. et Jacq.

Trois faisaient partie des collections de la "Southern Cross " étudiées par M. E.-A. Smith en 1902 : Levilittorina antaretica Sm., Rissoa adarensis Sm., Pecten Colbecki Sm.

Quatre ont été décrites, en 1903, par M. Pelsencer dans ses recherches ze Expédition Charcot, - Lır., - Gastropodes prosobranches, Scaphopode et Pélécypodes. 1 
sur les Mollusques recueillis par la "Belgica ": C'yamium subquendratum

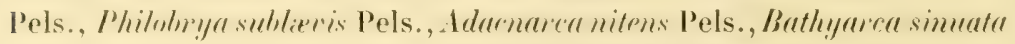
Pels.

Quatre étaient parmi les espèces nouvelles que j’ai fait connaitre, en 1906, dans les récoltes de la première expédition du D' Chareot: I'alvatella antarctica, Cyamiomactra laminifera, Montaguia Charcoti, Philobrya wandelensis.

Une a été signalée également par moi, en 1906, comme se rencontrant aux Oreades du Sud: Yoldia inrquisculpta.

Trois, trouvées par l'Expédition de la "Discovery ", ont été nommées par 1I. E.-A. Smith en 1907: Valuatella mimutissima Sm., Cyamium denticulatum Sm., Kellya simulans $\mathrm{Sm}$.

Lne est trìs probablement la mìme coquille que MII. Melvill et Standen ont identifire, en 190\%, dans les collections faites par l'Expédition du "Scotia ", au Dentalium Shoplandi Jouss.

Les huit espèces nouvelles sont : Buccinum Charcoti, Sipho Gaini, Ceri-

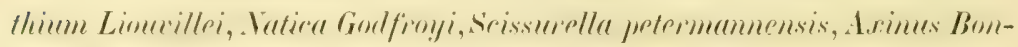
graini, Bathyarea Gourdoni, Silicula Rouchi. 


\begin{tabular}{|c|c|c|c|c|c|c|c|c|c|c|}
\hline & 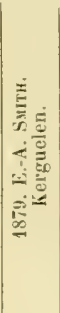 & 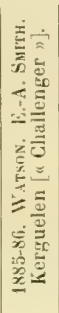 & 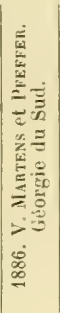 & 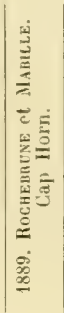 & 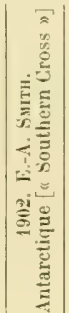 & 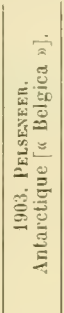 & 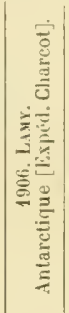 & 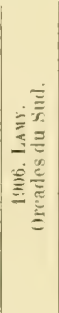 & 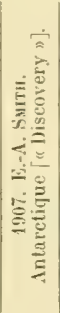 & 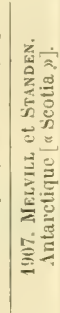 \\
\hline 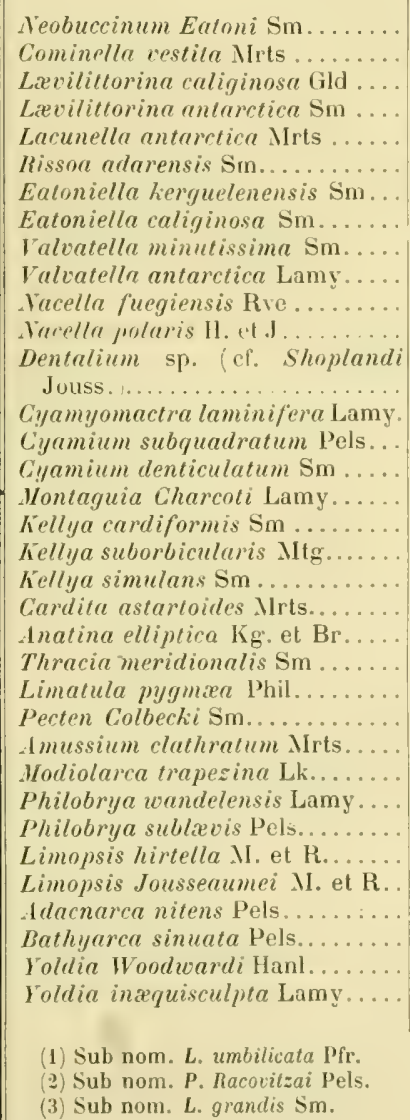 & $\begin{array}{l}+ \\
+\end{array}$ & $\begin{array}{l}+ \\
+ \\
+ \\
+ \\
+\end{array}$ & $\begin{array}{l}+ \\
+ \\
+\end{array}$ & + & $\begin{array}{l}+ \\
+ \\
+ \\
+\end{array}$ & $\begin{array}{c}+(2) \\
+ \\
+ \\
+ \\
+ \\
+\end{array}$ & $\begin{array}{c}+ \\
+ \\
+(11 \\
+ \\
+ \\
+ \\
+ \\
+ \\
+ \\
+ \\
+\end{array}$ & $\begin{array}{l}+ \\
+\end{array}$ & $\begin{array}{l}+ \\
+ \\
+ \\
+ \\
+\end{array}$ & $\begin{array}{l}+ \\
+ \\
+ \\
+ \\
+ \\
+ \\
+\end{array}$ \\
\hline
\end{tabular}




\section{GASTROPODES PROSORRANGIES. \\ Genre BUCC/NUM Linné, 1767. \\ Buccinem Chancoti Lamy.}

(Pl. I, fig. 1-2.)

1910. Buccinum Charcoti L.AyY, Gastrop. Miss. Charcot, Bull. Mus. Hist. Nal., t. XVI, p. :318.

Testa globoso-ovata, temuis, albida, epidermide griseo-lutescente induta. Spira brevis. Anfr. $41 / 2$ conrexi, sutura impressa et canaliculata sejuncti, rapide crescentes, striis spiralibus, temeibus, crehris, ornati; in anfr. ultimo permagno, inflato, $4 / 5$ totius longitudinis equante, strix incrementi longitudimales accedunt. Apertura margna, ablongo-ocata; columella arcuata, callo temui, lato, aduato; labrum acutum; canalis brevis, emarginatus.

Alt.: $33^{\mathrm{mm}}$; diam. max. : $20^{\mathrm{mm}}$. Apertura $95^{\mathrm{mm}}$ alta, $10^{\mathrm{mm}}$ lata.

Coquille de forme ovale, un peu globuleuse, à test mince; de couleur hanchàtre sous un épiderme gris jaunitre. spire courte, composée de quatre tours et demi, convexes, séparés par une suture bien marquée et canaliculée, croissant rapidement, ornés de stries transverses fines et serrées, qui, sur le dernier tour, très grand, renflé, formant à lui seul les $4:$ de la coquille, s'entre-croisent avec de nombreuses stries d'aceroissement. Ouverture grande, de forme ovale allongée; columelle arquée, couverte d'une très mince callosité appliquée, largement développée; labre tranchant; canal court, réduit à une échancrure.

$\mathrm{N}^{\circ}$ 747. - Milieu de la baie de l'Amirauté, île du Roi-George, Shetlands du sud, dragage XVII, $\$ 20$ mètres (26 décembre 1909): deux individus.

Les dimensions données ci-dessus se rapportent au plus grand des spécimens (P.I, I, fig. 1-2); l'autre a seulement 2 ' millimètres de longueur et 1 i millimetres de diametre maximum. Tous deux ne montrent aucune trace d'opercule.

Cette forme ne peut ćtre comparée qu'à des espèces des mers boréales : par son contour, elle oflre une certaine ressemblance avec le Volutharpa 
Mörchiana P. Fischer (1), des côtes de Sibérie, qui, d'après M. Wm. H. Dall (2), est une variété à spire courte du Buccinum cyaneum Brug. ; mais elle rappelle surtout le Buce. Fischeriunum Dall (3), de la mer de Behring.

\section{Buccinum sp. forma juvenilis.}

(Pl. I, fig. 3-4.)

$\mathrm{N}^{\circ}$ 714. - Anse onest de la baie de l'Amirauté, île du Roi-George, Shetlands du Sud, dragage XVIII, 7̈̈ mètres (27 décembre 1909): 1 individu.

Le dragage XVIII, effectué également dans la baie de l'Amirauté, a fourni une petite coquille composée de deux tours et demi de spire, ayant pour dimensions : hauteur, 6 millimètres; diamètre maximum, 3 millimetres. C'est très vraisemblablement la forme jeune d'un Buccin, et l'existence, à la face externe du labre, de plusieurs stries spirales tendrait à faire supposer qu’elle appartient à l'espèce précédente, trouvée dans la mème localité; mais on ne peut se prononcer arec certitude, cette coquille, qui, elle, est pourvue d'un opercule, étant unique et ayant été rencontrée isolément.

\section{Genre NEOBUCCINUM/ E.-A. Smith, 1879.}

\section{Neobuccinum Eatoni E.-A. Smith.}

187. Buccinopsis Ealoni E.-A. Smтr, Shells Kerguelen. Ann. Mag. Nal. Hist., te sér., vol. XVI, p. 68.

1879. Neobuccinum Ealoni E.-A. Smrtu, Moll. Kerguelen, Phil. Trans. Roy. Soc. London, vol. CLXVIII, p. 169, PI. IX, fig. 1-1 $a$.

1886. N. Ealoni Sm., Watson, Rep. "Challenger», Gasterop., p. 216.

1902. N. Ealoni E.-A. Smru, Voy. "Southern Cross ", Moll., p. 202.

1903. N. Ealoni Sm., v. MArtens, Deutsch. Tiefsee Exped. "Valdivia ", Gastrop., p. 63.

1906. N. Eatoni Sm., Lamy, Expéd. Antarct. Franc. du Dr Charcol, Gastrop., p. 2.

1907. N. Ealoni E.-A. Sмiтr, Nation. Anlarct. Exped. "Discovery ", Gastrop., p. 1.

1907. N. Ealoni Sm., Melvill et Standex, Scollish Nalion. Antarct. Exped., Moll.,

Trans. Roy. Soc, Edinburgh, vol. XLVI, p. 139.

1910. N. Ealoni Sm., Lamy, Moll. Kerguelen, Bull. Mus. Hist. Nat., t. XVI, p. 199.

(1) 1839, Journ. de Conchyl., vol. VII, p. 299, PI. X, fig. $2 a-b$.

(2) 1872, Americ. Journ. of Conchol., vol. VII, P. 103.

(3) 1872 , itid, p. 106, P!. XII, fig. 13. 
No 2'. - Chenal Peltier, entre l'ilôt Goetschy et l'ile Doumer, dragage $\mathrm{V}, 92$ mètres (29 décembre 1908) : I individu.

$X^{\text {Tos }} 766$ et $7 \%$. Milieu de la baie de l'Amirauté, île du Roi-George, Shellands du Sud, dragage XVII (26 décembre 1909) : 11 individus.

No 847. - Par $70^{\circ} 10^{\prime}$ latitude sud et $80^{\circ} 30^{\prime}$ longitude ouest, en bor'dure de la banquise, dragage $X X, 460$ mètres $(12$ janvier 1910): 1 individu.

Genre COMINELLA Gray, 184.

Cominella (Chlanidota) vestita v. Martens var. elongata Lamy.

(PI. I, fig. 6.)

1878. Cominella veslila v. Martens, Silzungsber. Ges. Nalurf. Fr. Berlin, p. 23.

1880. Buccinum (Chlanidola) veslilum v. Martens, Conchol. Millheil., I, p. 43, PI. IX,

fig. $3 a-c$.

1886. Neobuccinum veslilum MIrts., Watson, Rep. "Challenger ", Gasterop., p. 216.

1902. Chlanidola vestila Mrts., E.-A. Swith, Voy. "Southern Cross ", Moll., p. 203.

1903. Cominella (Chlanidola) vestila v. Martens, Deulsch. Tiefsee-Exped. "Valdivia", Gastrop., p. 63.

1910. C. (Chlanidola) vestila Mrts., LAMy, Moll. Kerguelen, Bull. Mus. Hisl. Nal., t. XVI, 1. 1999.

1910. C. (Chlanidola) vestila Mrts. var. elongala LAMY, Gastrop. Miss. Charcot, Bull.

Mus. Hist. Nal., t. XVI, p. 319.

No 717. - Nilieu de la baie de l'Amirauté, île du Roi-George, Shetlands du Sud, dragage XVI, 420 mètres (26 décembre 1909): 1 individu.

Cette coquille est, par l'ensemble de ses caractères, identique au C. cestitu v. Martens, de Kerguelen, dont j'ai pu récemment examines plusieurs individus provenant des récoltes faites en 1909 dans cette localité par M. Rallier du Baty; mais elle est beaucoup plus allongée : en effel, tandis que deux spécimens, dont v. Martens (1880 et 1903) a donné les dimensions, larges l'un de 16 millimètres, l'autre de 21 millimètres, avaient pour longueur le premier 23 millimètres, le second 29 millimètres, lexemplaire recueilli par 11 . Lionville a, pour une largeu de 17 millimètres, une longueur de 30 millimètres; il a, par suite, un contour ovale (PI. I, fig. 6), tout différent de l'aspect globuleux de la 
forme typique (Pl. I, fig. :̈), à laquelle je le rattache comme constituant une variété elongatı.

\author{
Genre SIPI/O Klein, 1733. \\ Sipho Gaini Lamy. \\ (PI. I, fig. 7-8.)
}

1910. Sipho Gaini Lair;, Gastrop. Miss. Charcot, Bull. Mus. Hist. Nal., t. XVI, p. 319.

Testa fusiformis, temuis, albida, epidermide tenuissima lutescente induta. Spira turrita. Anfi. 5 convexinsculi, regulariter crescentes, sutura canaliculata sejuncti, spiraliter confertim lirati. In anfir. ultimo marno, tester dimidiam partem requante, strix incrementi longitudinales accedunt. Apertura anguste ovata, inferme in canalem obliqum brevem apertum desinens; columella arcuata, callosa; labrum acutum. Operculum unguiforme, corneum, lutescens, mucleo apicali.

Alt.: $33^{\mathrm{mm}}$; diam. max. : 12 $2^{\mathrm{mm}}$. Apertura $19^{\mathrm{mm}}$ altu, $6^{\mathrm{mm}}$ latu.

Coquille fusiforme, a test mince, de couleur blanchatre sous un épiderme. jaunitre trìs mince. Spire turriculée, composéc de cing tours légierement convexes, à croissance régulière, séparés par une suture canaliculéc, ornés de cordons décurrents nombreux et serrés, qui, sur le dernier tour égal à la moitié de la hauteur totale de la coquille, sont croisés par des stries d'accroissement. Ouverture ovale, étroite, se terminant, dans le bas, par un court canal, oblique, ouvert; columelle arquée, pourvue d'une callosité; labre tranchant. Opercule ongaiculé, corné, jaunitre, à nucléus apical.

$N^{0.747 .}$ - Milieu de la baie de l'Amirauté, île du Roi-George, Shetlands du Sud, dragage YVII, 120 mètres 26 décembre 1909): 1 individu.

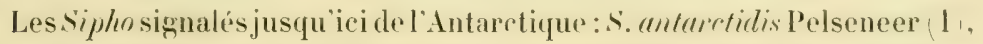
Troschelia ou Siphosp. E. -A. Smith (2), S. archibenthalis et S. crassicostatus Melvill et Standen (3), ont tous une forme plus trapue et une ouverture bien moins allongée.

(1) 1903, Voy. "Belgica ", Moll., p. 22, Pl. V, fig. 60.

(2) 1!107, Nation. Antarct. Exped. "Discovery ", Gastrop., p. 2, Pl. I, fig. 8.

(3) 1907, Scottish Yation. Anturct. Exped., Moll., Trans. Roy. Soc. Edinburgh, vol. XLVI, p. 138, Pl. 1, fig. 9 et 10 . 


\section{Genre CERITHIUM Adanson, $173 \%$.}

Cenithium Liouviller Lamy.

(Pl. I, fig. 9.)

1910. Cerilhium Liouvillei Lamy, Gastrop. Miss. Charcot, Bull. Mus, Hisl. Nal., t. XVI, p. 320 .

Testa parea, comica, lutescens. spira clata, turrita, acuminata. Anfir. 101 : comexi, sutura impressa sejuncti, longitudinaliter costis tenubus, spiratiter earinis duabus valde eminentibus ormati; funiculus humilior suturam obtegit et in anfir. ultimo basim, funiculis duobus aliis munitam, cingit. Apertura rotundato-ovata, infra canaliculata; columella arcuata; labrum acutum; canalis brevis.

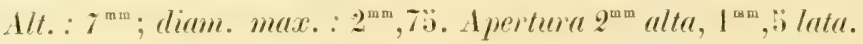

Coquille petite, conique, jaunàtre. Spire élevée, turriculée, aiguë, comprenant dix tours et demi, séparés par une suture bien marquée, ornés longitudinalement de fines côtes et transversalement de deux carènes très saillantes; un cordon plus faible recouve la suture et, dans le dernier tour, circonscrit la base, qui est munie de deux autres cordons. (Huverture ovale-arrondie, canaliculée inférieurement; columelle arquér; labre tranchant; canal court.

To 649. - Port-Lockroy, chenal de Rosen, dragage IV, 70 mètres 26 novembre 1909): I individu.

Ce Cérithe diftère du C'. Charcoti Lamy (I) par sa forme plus allongée et par l'existence, sur chaque tour, de deux carènes, au lieu de trois cordons prineipaux. Par sa seulpture, il se rapproche plutot du C: georgianum Plfr. (2), mais il est bien plus conique, avec une spire plus aiguë, ct ses tour's, plus nombreus, sont plus trapus, surtout le dernier, qui est relativement tè̀s court.

Genre LEVILITTORINA Pfefier, 1886.

Lisvilittorina caliginosa Gould.

1849. Lillorina caliginosa Gould, Proc. Boslon Soc. Nal. Hist., III, p. 83.

1) 1906, Expeil. Antaret. Franc. du br Charcot, Gastrop., p. 4, Pl. 1, fig. 1.

(2) $1886, x$. Mirtexs el Prafrer, Moll. Siid-Georgien, Jithrb. Himburg. Wiss. Inst., III, p. 97, Pl. 11, fig. 7 . 
1852-56. L. caliginosa Gould, U. S. Explor. Exp. Wilkes, p. 198, Pl. XIV, fig. 240.

1879. Hydrobia caliginosa Gld., E.-A. Surth, Moll. Kerguelen, Phil. Trans. Roy. Soc. London, vol. CLXVIII, p. 173, Pl. IX, fig. 8.

1886. H. caliginosa Gld., Watson, Rep. "Challenger ", Gasterop., p. 613.

1886. Lavilittorina caliginosa Gld,, v. Martens et Pfeffer, Moll. Süd-Georgien, - Jahrb. Hamburg. Wiss. Anst., III, p. 81, PI. I, fig. 8 a-d.

1889. Paludestrina caliginosa Gld., De Rochebrune et Mabille, Miss. Scient. Cap

Horn, Moll., p. 42.

1903. Lævilillorina caliginosa GId., Pelseneer, Voy. "Belgica », Moll., p. 8.

1906. L. caliginosa Gld., Lamy, Moll. Orcades du Sud, Bull. Mus. Hist. Nat., t. XII, p. 11:?

1906. L. caliginosa Gld., Lams, Expéd. Anlarct. Franç. du Dr Charcol, Gastrop., p. 4. 1907. L. caliginosa Gld., H. Streber, Moll. Magalhaen., Zool. Jahrb., vol. XXV, p. 156. 1907. Lillorina (Lævilillorina) caliginosa Gld., Melvill et Standen, Scollish Nation.

Anlarcl. Exped., Moll., Trans. Roy. Soc. Edinburgh, vol. XLVI, p. 130.

1911. Levilitlorina caliginosa Gld., Lamy, Moll. Géorgie du Sud, Bull. Mus. Hist. Nat.,

t. XVII, p. 22.

Nos 1.2 et 194. - Ile Wandel, cote sud ( 16 février 1909): 60 individus.

No 302. - Ile Petermann, anse nord (10 octobre 1909): 3 individus.

No 3.1. - Ile Petermann (29 octobre 1909): 2 individus.

No 3̈38. - Ile Petermann, plage est (novembre 1909): 1 individu.

No 624. - Ile Petermann, nord (30 octobre 1909): 2 individus.

No 689. - Baie de l'Amirauté, ile du Roi-George, Shetlands du Sud (26 décembre 1909 ) : 0 individus.

No 736. - Port-Lockroy (janvier 1910) : 12 individus.

Lævilittorina antarctica E.-A. Smith.

1902. Paludestrina antarctica E.-A. Sumtr, Voy. "Southern Cross», Moll., p. 204, Pl. XXIV, fig. 16.

1906. Lævilitlorina umbilicala Lamy (non Pfeffer), Expéd. Antarct. Franç. du Dr Charcol, Gastrop., p. 5.

No 132. - Iles Argentines (8 février 1909): 50 individus.

No 142. - Ile Wandel, còte sud (16 février 1909): 20 individus.

$N^{\text {os }} 302$ et 312 . - Ile Petermann, anse nord (10 octobre 1909): 40 individus.

No 325. - Ile Petermann, nord-est (16 octobre 1909): 2 individus.

No 328. - Ile Petermann (21 octobre 1909): I individu.

Nos 334 et 347 . - Ile Petermann (29 octobre 1909): 3 individus.

No 366 . - Ile Petermann: 10 individus.

Dans mon travail sur les Mollusques de la première expédition du 20 Expédition Charcol. - L.MY. - Gastropodes prosobranches, Scaphopode et Pélécypodes. 2 
$D^{r}$ Charcot, j'avais rapporté, d'ailleurs, avec un certain doute, au Lavilittorina umbilicata Pffr. (1) des coquilles qui, d'une part, se distinguairnt du Lipe. raligimess Cild. par leur spire beaucoup plus aignü, leurs tours arrondis et étagés, leur ouverture presque circulaire, mais chez qui, d'autre part, les carènes et la fossette ombilicale mentionnées par P'feffer pour son espèce n'étaient que très faiblement indiquées. L'examen que j'ai fait de nouveaux spécimens entièrement semblables recueillis par M. le Dr Liouville m'a démontré que ces coquilles correspondent bien plus exactement à la forme décrite en 1902 par M. E.-A. Smith sous le nom de Paludestrima anturctica. Je les identifie dono actuellement à cette dernière espèce plutôt qu'au $L$. umbilicuta. Je ferai remarquer toutefois que l'appellation spécifique choisie par II. Smith est assez fàcheuse, car elle pourrait prêter ì confusion : antérieurement à son Paludestrina antarctica, il existait déjà, en effet, un Iydrobia antarctica Philippi (2).

\section{Genre LACUNELLA Dall, 1884. \\ Lacunella Antarctica vo Martens.}

1885. Lacuna antarclica v. Martens, Moll. Süd-Georgien, Silzungsb. Ges. Nalurf. Fr. Berlin, p. 92.

1886. Lacunella anlarclica Mrts., v. Mantens et Pfeffer, Moll. Süd-Georgien, Jahrb.

Hamburg. Wiss. Anst., III, p. 89, Pl. II, fig. 1 a-f.

1906. L. antarctica Mrts., LAmy, Moll. Orcades du Sud, Bull. Mus, Hist. Nal., t. XII, p. 123.

1906. L. anlarclica Mrts., Lamy, Expéd. Anlarcl. Franç. du Dr Charcot, Gastrop., p. 6

No 689. - Baie de l'Amirauté, île du Roi-George, Shetlands du Sud (26 décembre 1909): 2 individus.

Genre RISSOA Fréminville, 1814.

Rissoa adarensis E.-A. Smith.

1902. Rissoa adarensis E.-A. Sмiтн, Voy. "Southern Cross ", Moll., p. 205, Pl. XXIV, fig. 17 .

(1) 1886, v. Martens et P'feffer, Moll. Sül-Georgien, Jahrb. Hamburg. Wiss. Anst., III, p. 88, P'1. 1, fig. 12.

(2) 1868, Malak, Blät., vol. XV, p. 2?'́, - 1903, Pelseneer, Voy. "Belgica ", Moll., p. 8. 
1907. R. alarensis E.-A. Smitr, Nalion. Anlarcl. Exped. "Discovery ", Gastrop., p. 8,

Pl. II, fig. 2.

1907. R. adarensis Sm., Melvill et Standen, Scollish Nation. Antarcl. Exped., Moll., Trans. Roy. Soc. Edinburgh, vol. XLVI, p. 132.

No $312 .-$ Ile Petermann, anse nord (10 octobre 1909) : 1 individu.

Ce spécimen correspond bien à la première figure donnée pour cette espèce par M. E.-A. Smith (1902).

Genre EATOVIELLA Dall, 1876.

Eatoniella herguelenensis E.-A. Smith.

1875. Ealonia kerguelenensis E.-A. Sмгтн, Shells Kerguelen, Ann. Mag. Nal. Hist., 4e sér., vol. XVI, p. 70.

1876. Eatoniella kerguelenensis Sm., DaLL, in Krdder, Nat. Hist. Kerguelen, Bull. U. S. Nat. Mus., III, p. 42.

1879. E. kerguelenensis E.-A. Sмтн, Moll. Kerguelen, Phil. Trans. Roy. Soc, London, vol. CLXVIII, p. 174, Pl. IX, fig. 10.

1886. E. kerguelenensis Sm.,v, Martens et PfefFer, Moll. Süd-Georgien, Jahrb. Hamburg. Wiss. Anst., III, p. 94, Pl. II, fig. 5.

1906. E. kerguelenensis Sm., LAmy, Expéd. Antarct. Franç. du Dr Gharcol, Gastrop., p. 7.

No 312. - Ile Petermann, anse nord (10 octobre 1909): 4 individus.

No 317 . - Ile Petermann (29 octobre 1909): 2 individus.

Eatoniella caliginosa E.-A. Smith.

1875. Ealonia caliginosa E.-A. Smitr, Shells Kerguelen, Ann, Mag. Nal. Hist., 4e sér,, vol. XVI, p. 71.

1876. Eatoniella caliginosa Sm., DAl, in Kidder, Nat. Hist. Kerguelen, Bull. U. S. Nat. Mus., III, p. 43.

1879. E. caliginosa E.-A. Sмiтн, Moll. Kerguelen, Phil. Trans. Roy. Soc. London, vol, CLXVIII, p. 175, PI. IX, fig. 9.

1886. E. caliginosa Sm., Watson, Rep.. "Challenger", Gasterop., p. 614.

1906. E. caliginosa Sm., LAMY, Expéd. Anlarct. Franç. du $D^{\mathrm{r}}$ Charcol, Gastrop., p. 7.

No 271. - Ile Petermann, còte nord-est, chenal de Lemaire, dragage XIV c, 60 à 40 mètres (18 novembre 1909): 1 individu.

No 302. - Ile Petermann, anse nord (10 octobre 1909): 10 individus.

No 736. - Port-Lockroy (janvier 1910): 7 individus. 
Genre NATHA Adanson, 1757.

Natica Godfroyi Lamy.

(Pl. I, fig. 10-11.)

1910. Nalica Godfroyi Layr, Gastrop. Miss, Charcot, Bull. Mus. Hist. Nat., t. XVI, p. 322 .

Testa subglobosa, lavis, albida, cpidermide olivaceo-fusca induta. Spira lrevissima. Anfi. i concexi, superne subdepressi, rapide crescentes, sutura impressa ac marginata sejuncti. Apertura ovata, superne angulata, inferne rotundata; columella callo umbilicum ommino obtegente munita; labrum arcuatum, simplex.

Att. : $12^{\mathrm{mm}}$; diam. max. : $11^{\mathrm{mm}}$. Apertura $9^{\mathrm{mm}}$ alta, $6^{\mathrm{mm}}$ latu.

Coquille presque globuleuse, lisse, blanchàtre, recouverte d'un épiderme brun olivâtre. Spire très courte, composée de quatre tours convexes, légèrement déprimés en dessus, croissant très rapidement, séparés par une suture bien marquée, au-dessous de laquelle il y a un léger bourrelet. Ouverture ovale, anguleuse en haut, arrondie en bas; columelle pourve d'une callosité fermant entièrement l'ombilic; labre arqué, simple.

N. 747. - Nilieu de la baie de l'Amirauté, île du Roi-George, Shetlands du sud, dragage XVII, 120 mètres 126 décembre 1909): 1 individu.

Cette Natice rappelle beaucoup par sa forme le $\mathrm{N}$. consolidata Couthouy (1), de la cote atlantique des Etats-Lnis, que Philippi (2) rattache comme variété minor au $N$. clausa Brod. et Sow.

Parmi les espèces des mers australes, sans ombilic et à spire très courte, le $\boldsymbol{N}$. impervia Philippi $(3)$, du détroit de Magellan, auquel M. II. Strebel (4) identifie avec raison le N. Payeni Rochebrune et Iabille (:3), est de contour bien plus ovalaire; Ie. I. furtilis Watson (6), des iles Kerguelen, Marion, Prince-Edouard, a une spire plus haute, les tours

(1) 1839, Boston Journ. Nat. Hist., vol. II, p. 89, Jl. III, fig. I4.

(2) 1852, Martixi u. Cuemsixz, Conch. Cab., ₹e édit., Natica, P. 99, Pl. X1Y, fig. 6.

(3) 1845, Abild. Conch, vol. II, p. 42, Pl. II, fig. 6.

(4) 1907, Moll. Magalhaen., Zool. Jahrb., vol. XXIV, p. 135.

(5) 1889, II iss. Scient. Cap Horn, Moll., p. 32, Pl. III, fig. 6.

(6) 1886, Rep. "Challenger ", Gasterop., p.446, PI. XXVII, fig. 10. 
étant moins déprimés; le $N$. prasina Watson (1), de Kerguelen, se distingue par- l'absence de bourrelet au-dessous de la suture et par le fait que les tours ne sont pas déprimés supérieurement.

\section{Genre VALVATELLA Gray, 1837. \\ Valvatella Minutissima E.-A. Smith.}

1907. Valvalella minulissima E.-A. Sмттн, Nalion. Antarcl. Exped. "Discovery ", Gastrop., p. 12, Pl. II, fig. 10 .

Yo 286. - Près de la Terre Nlexandre- $I^{\mathrm{e}}$, dragage $\mathrm{X}, 297$ mètres (22 janvier 1909): 1 individu.

Je rapporte à cette espèce une petite coquille turbinée, haute de 4 millimetres, composie de quatre tour's et demi, pourve d'une perforation ombilicale extrèmement étroite ef présentant quelgues ragues indices de striation spirale.

\section{Valvatella antarctica Lamy.}

1905. Margarila anlarctica LAMY, Gastrop. Expéd. Charcot, Bull. Mus. Hist. Nal., t. XI, p. 481.

1906. M. anlarctica Lamy, Moll. Orcades du Sud, Bull. Mus. Hist. Nat., t. XII, p. 123. 1906. M. anlarctica LAмx, Expéd. Antarct. Franç. du $D^{\mathrm{r}}$ Charcol, Gastrop., p. 9, Pl. I, fig. $2,3,4$.

1907. Valvalella anlarctica Lamy, Melvill et Standen, Scollish Nation. Anlarct. Exped., Moll., Trans. Roy. Soc. Edinburgh, vol. XLVI, p. 129.

1911. V. anlarctica LAMY, Moll. Sandwich du Sud, Bull. Mus. Hist. Nal., t, AVII, p. 25.

Nos 123 et 124, - Ile Petermann (11 février 1909): 2 individus (dans l'estomac d'un Poisson).

No 138. - Ile Petermann (février 1909) : 4 individus.

Yo 271. - Ile Petermann, còte nord-est, chenal de Lemaire, dragage XIV $c, 60$ à 40 mètres (18 novembre 1909): 20 individus.

No 300 . - Ile Petermann (2 octobre 1909): 11 individus.

$\mathrm{N}^{\mathrm{os}} 307,308$ et 312 . - Ile Petermann, anse nord ( 10 octobre 1909):

7 individus.

No $32 \%$. - lle Petermann, nord-est (16 octobre 1909): 1 individu.

Yos 330 et 452 . - Ile Petermann : 1: individus.

(1) 1886, ibid., p. 449, [']. XXVII, fig. 9. 
No 626. - Ile Petermann, cote nord-est, chenal de Lemaire, dragage XIII b, 30 à l: mètres (17 novembre 1909): 1 individu.

To 629. - Ile Petermann : 2 individus.

No 689. - Baie de l'Amirauté, île du Roi-George, Shetlands du Sud (26 décembre 1909): 2 individus.

No 803 . - Par $70^{\circ} 10^{\prime}$ latitude sud et $80^{\circ} 50^{\prime}$ longitude ouest, en bordure de la banquise, dragage XX, 460 mètres ( 12 janvier 1910): 6 individus.

Cette espèce, que j’ai décrite comme nouvelle parmi les Gastropodes rapportés par la première expédition du $\mathrm{D}^{\mathrm{r}}$ Charcot et qui est bien caractérisée par sa coquille orbiculaire, obtuso-conique, profondément ombiliquée, de coloration gris-bleuàtre ou verdâtre, a été retrourée ultérieurement aux Oreades du Sud, à la fois dans les récoltes de M. Valette Lamy, 1906i) et dans celles de l'expédition antarctique écossaisse (Melvill et Standen, 1907), puis tout dernièrement aux îles Sandwich du Sud (Lamy, 1911).

\section{VAlvatella sp. forma juvenilis.}

(Pl. I, fig. 12.)

No $312 .-$ Ile Petermann, anse nord (10 octobre 1909): 1 individu.

11. le $\mathrm{D}^{\mathrm{r}}$ Liouville a recueilli à l'ìle P'etermann une petite coquille orbiculaire et déprimée, dont le diamètre n'est que de 1 millimètre et la hauteur de $0^{\mathrm{mm}}, 73$; elle est composée de trois tours dont le dernier, profondément ombiliqué, présente deux carènes spirales aiguïs (Pl. I, fig. 12) : elle ne montre pas trace de cotes longitudinales et, par leur absener, elle differe done d'une forme minuscule de mème aspect (PI. I, fig. 13) trouvée par II. Valette en 1904 aux Orcades du Sud, que j'ai décrite en 1906 (1) et dont j’ai sisnalé la grande ressemblance avec le jeune du .Yargarita cimeren Couthouy, des mers septentrionales d'Europe et d'Amérique, tel qu'il a été figuré par G. O. Sars (2). Mais la coquille rapportée par M. Liouville ayant été récoltée seule, ainsi que celle de M. Valette, il est également impossibtr de les rattacher avec certitude comme formes jeunes, l'une ou l'autre, à tel ou tel Valvatella (= Margarita $)$ des mers australes.

(1) 1906, Bull. Mus. Hist. Nat., t. Xll, p. 123.

(2) 1878, Moll. Reg. Arctic. Norvegix, p. 135, Pl. XXl, fig. 1. 
Genre NACELLA Schumacher, 1817.

Nacella (Patinella) fuegiensis Reeve.

1855. Palella fuegiensis Reeve, Conch. Icon., VIII, Patella, Pl. XXVIII, fig. $73 a-b$.

1879. P. (Patinella) fuegiensis Rve., E.-A. Smitr, Moll. Kerguelen, Phil. Trans, Roy.

Soc. London, vol. CLXVIII, p. 180, Pl. IX, fig. 14-14a.

1886. P. fuegiensis Rve., IVAtson, Rep. "Challenger ", Gasterop., p. 26.

1889. P. fuegiensis Rve, de Rochebrune et Mabille, Miss. Scient. Cap Horn., Moll., p. 95 .

1903. P. (Palinella) fuegiensis Rve., v. Martens, Deulsch. Tiefsee-Exped. "Valdivia", Gastrop., p. 72.

1907. P. fuegiensis Rve., Melvill et Standen, Scottish Nalion. Anlarcl. Exped., Moll., Trans. Roy. Soc. Edinburgh, vol. XLVI, p. 127.

1910. Nacella (Palinella) fuegiensis Rve., LAмy, Moll. Kerguelen, Bull. Mus, Hist. Nat., t. XVI, p. 20 ?

No 308. - Ile Petermann, anse nord $(10$ octobre 1909) : 4 individus. Nacella (Patinella) polaris Hombron et Jacquinot.

1841. Palella polaris Hombron et Jacquinot, Moll. "Astrolabe " et "Zélée », Ann. Sc.

Nat. Zool., 2e sér., vol. XVI, p. 191.

1886. $P$. polaris Hombr, et Jacq., v. Martens et Pfeffer, Moll. Süd-Georgien, Jahrb.

Hamburg. Wiss. Ansl., III, p. 101, Pl. II, fig. 11-13.

1903. Nacella ænea Martyn var. polaris H, et J., Pelseneer, Voy. "Belgica ", Moll., p. 14.

1906. N. (Patinella) polaris H. et J., LAMr, Expéd. Anlarct. Franç.du Dr Charcol, Gastrop., p. 10.

1907. Palella polaris H. et J., Melvill et Standen, Scollish Nalion. Anlarcl. Expéd., Moll., Trans. Roy. Soc. Edinburgh, vol. XLVI, p. 127.

1911. Nacella (Patinella) polaris H. et J., LAmy, Moll. Géorgie du Sud, Bull. Mus. Hist. Nat., t. XVII, p. 26.

Port-Lockroy (29 décembre 1908) : 2 individus.

Baie Marguerite, entre l’ìle Jenny et la Terre Adélaïde (janvier 1!)0!! :

60 coquilles.

No 302. - Ile Petermann, anse nord (10 octobre 1909): 1 jeune individu.

No 334. - Ile Petermann : 2 individus.

No 689. - Baie de l'Amirauté, île du Roi-George, Shetlands du Sud (26 décembre 1909) : 1 jeune individu. 
Genre SCISSURELLA A. d'Orbigny, 1823.

Scissurella petermannensis Lamy.

(Pl. I, fig. 14, 15, 16.)

1910. Scissurella pelermannensis Lam, Gastrop., Miss. Charcot, Bull. Mus. Hist. Nal., t. XVI, p. 323 .

Testa mimutissima, temuissima, albida. Spira brevis, depressa. Anfi. 3, rapide crescentes, gradati. Anfr. ultimus, permaymus, lamellis calde eminentitues, distantibus ormatus, superne complanatus, inferne convexus ac vrofunde umbilicatus. A pertura maxima, oblique, subquedrangularis; columella paululum arcuata, margine leviter supra umbilicum reflexo instructa; Iabrum arcuatum, acutum, superne scissura angusta, qux lumella elevata utrinque marginata est, divisum.

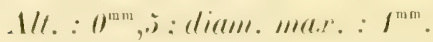

Coquille très petite, très mince, blanchàtre. Spire courte, déprimée, composée de trois tours étagés s'accroissant rapidement. Dernier tour très grand, muni de côtes lamelleuses fort élevées et espacées, plan à sa parlie supérieure, convexe à la base et profondément ombiliqué. Ouverture très grande, oblique, subquadrangulaire. Columelle un peu arquée, pourvue d’un bord qui se réfléchit légèrenent sur l'ombilic. Labre arqué, tranchant, fendu à sa partie supérieure par une scissure étroite bordée de deux lamelles élevées.

No 312. - Ile Petermann, anse nord (10 octobre 1909): 1 individu.

Cette minuscule coquille est bien caractérisée par ses côtes lamelleuses saillantes : parmi les formes vivantes figurées par les auteurs, la seule oflrant quelque ressemblance à ce point de vue est le Scissurella coronata Watson (1), de Tahiti :

\section{SCAPIIOPODE}

Genre IIENTALIUI/ Linné, 1738.

Dentaliuis sp. (ef. D. Shoplandi Jousseaume).

1894. Denlalium Shoplandi Jousseaume, Bull. Soc. Philom. Paris, 8 e sér., t. VI, p. 102,

1) 1886, Rep. "Challenger ", Gastrop., p. 11'k, Pl. VIII, fig. \&. 
1907. D. Shoplandi Jouss, Melvill et Staxdex, Scollish Nation. Anlarcl. Exped., Moll.,

Trans. Roy. Soc. Edinburgh, vol. XLVI. p. 143.

No 119. - Baie Matha, dragage XI, 380 mètres (1 février 1909): 1 individu.

No 286. - Près de la Terre Alexandre-I ${ }^{\mathrm{r}}$, dragage X, 297 mètres (22 janvier 1909): 2 individus.

No 788. - Par $70^{\circ} 10^{\prime}$ latitude sud et $80^{\circ} 30^{\prime}$ longitude ouest, en bordure de la banquise, dragage XX, 160 metres $(12$ janvier 1910$): 1$ individu.

Ce Scaphopode est orné de côtes longitudinales nombreuses : par ce caractère, il rappelle le $D$. majorimm Mabille et Rochebrune (1), de la baie Orange; mais celui-ci en compte près du sommet une douzaine et à l'ouverture une trentaine, comme le dit M. Pilsbry (2), et ainsi que j'ai pu le vérifier sur le type de l'espèce conservé au Muséum de Paris; or la forme rapportée par $\mathbf{H}$. le $\mathrm{D}^{\mathrm{r}}$ Liouville en présente une vingtaine au sommet et $2: 3$ à l'ouverture; elle oflre done une ressemblance plus grande avec le $D$. Shoplandi Jouss., tel que 11 . Pilsbry (3) en a précisé la description : aussi est-ce très probablement elle que l'expédition écossaise a recueillie dans l'Antaretique et que $\mathbf{M}$.I. Melvill ef standen ont eru pouvoir réunir à ce $D$. Shoplandi; mais celui-ci est une espèce draguée à $\vdots 0$ milles d'Adern, et il s’agit là d’une diffrerener d'habitat si considérable qu'il est permis de conserver des doutes sur cette identification.

\section{PELLEGYPODES}

Genre AXINUS J. Sowerby, 1821.

Axinus Bongraini Lamy.

(Pl. I, fig. 17.)

1910. Axinus Bongraini Lamy, Pélécyp. Miss. Charcot, Bull. Mus. Hist. Nat., t. XVI, p. 389.

Testa parva, rotundato-trigona, inflata, temis, aquivalvis, inequilatoralis, striis concentricis ornata, plici suludorsali parum conspicua munita, alba, crusta ferruginusa antice el postice tecta. Latus anticum leviter expan-

(1) 1889, Mise. Scient. Cap Horn, Moll., p. 100, PI. IV, fig. 10.

(2) 1897, Tryox, Man. of Conch., vol. XVII, p. 27, Pl. XII, fig. 98-99.

(3) 1897, ibid., p. 28, Pl. Xll, fig. 100.

ge Expédition Charcot, - Lux. - Gastropodes prosobranches, Scaphopode el Pélécypodes. 
sum, rotundatum; latus posticum brevius, subungulatum. Umbones prominuli, antrorsum incurvati. Cardo edentulus; ligamentum partim intermum.

Dirm. ant.-post. $6^{\text {num }}$; diam. umbono-ventr. : $5^{\mathrm{mm}}, 5$; crass. : $4^{\mathrm{mm}}$.

Coquille petite, de contour triangulo-arrondi, globuleuse, mince, équivalve, inéquilatérale, ornée de stries concentriques, pourvue presque dorsalement d'un pli peu marquí. Couleurblanche; un enduit ferrugine recouvre les régions antéricure et postérieure. Côté antérieur légèrement dilatí el arrondi crité postérieur plus court et subanguleux. Sommets un peu saillants, incurvés en avant. Charnière dépourvue de dents; ligament en partie interne.

No $2.3 \%$ - Ile Petermann: 1 individu.

Cette forme est voisine de certains $A$ ximus des mers septentrionales: elle diflère de l'A. flexusus Mtg. (1) par son bord antérieur arrondi et non pas anguleux et de l'A. Sarsi Phil. (2) par son contour plus triangulaire.

\section{Genre CYAIIOMACTRA F. Bernard, 189\%. \\ Cyamomactra laminifera Lamy.}

1906. Mactra (Heleromactra) laminifera LAMY, Lamellibr. Expéd. Charcot, Bull. Mus. Hist. Nal., t. XII, p. 45.

1906. Cyamiomaclra laminifera Lamy, Moll. Orcades du Sud, Bull. Mus. Hist. Nat., t. XII, p. 121 (en note).

1906. C. laminifera LAıy, Expéd. Anlarcl. Franç. du Dr Charcol, Péléeyp., p. 11, Pl. I, fig. 10-11-12.

1911. G. laminifera Layy, Moll. Sandwich du Sud, Bull. Mus. Hist. Nal., t. XVII, p. 26.

Nos 123 et 133. - Ile Petermann (11 février 1909): 7 individus (dans l'estomac d'un Poisson).

No 172. - Ile Petermann (février 1909): 4 individus (dans l'estomac d'un Poisson).

No 689. - Baie de l'Amirauté, île du Roi-George, Shetlands du Sud (26 décembre 1909): 1 individu.

Cette espèce était une des formes nouvelles trouvées par la première expédition du Dr Charcot; depuis lors, le Muséun de Paris a reçu de

(1) 1803, Mostagu, Test. Britann., p. 72 [Tellina $]$ - 1878, G.-0. Sars, Moll. Reg. Aretic. Norvegix, p. 59, PI. XIX, fig. $4 a-b$.

(2) 1845 , Pulcipp1, Zeitschr:f. Malak., p.91. - 1878, G.-0. Sans, Moll. Reg. Arctic. Norvegix, P. 60, PI. XIX, fig. $5 a-6$ 
11. Lahille, en 1910, d'autres spécimens recueillis aux îles Sandwich du Sud.

\section{Genre CYLIIUM Philippi, 1845. \\ Cyamum subquadratum Pelseneer.}

1903. Cyamium subquadralum Pelseneer, Voy. "Belgica », Moll., p. 15, Pl. IX, fig. 124. 1906. C. subquadralum Pels., Lamy, Expéd. Antarct. Franç. du Dr Charcot, Pélécyp., p. 13.

No 132. - Iles Argentines (8 février 1909): 2 individus.

Yo 1'11. - lle Wandel, côte sud (16 février 1909): 200 individus.

No 323 . - Ile Pertermann, nord-est 16 octobre 1909 : 1 individu.

No 366. - Ile Petermann : 60 individus.

No 678. - Port-Lockroy (novembre 1909): 40 individus.

No 736. - Port-Lockroy (janvier 1910): 60 individus.

Cette espèce très inéquilatérale, à còté antéricur plus court et moins haut que le postérieur, est de coloration brun rougeâtre, tandis que le C. antarcticum Philippi (1), le C. fallilandicum Mlelvill et Standen (2) et le $C$. iridescens Preston (3) sont des coquilles oblongues, moins inéquilatérales, dont la couleur est blanche.

\section{Gyamum denticulatum E.-A. Smith.}

1907. Cyamium denliculalum E.-A. Sмiтн, Nation. Antarct. Exped. "Discovery", Lamellibr., p. 3, Pl. III, fig. 4-4 $b$.

No 673. - Port-Lockroy: 1 individu.

Cette petite coquille offre, en miniature, l'aspect d'un Cardium, et elle possède une charnière composée, dans la valve gauche, de trois dents, dont la médiane est bifide, et, dans la valve droite, de deux, dont l'antérieure est bifide: elle correspond donc parfaitement à la description donnée par M. E.-A. Smith pour son $C$. denticulatum.

\section{Genre HONTAGUIA Turton, 1822 (emend.). \\ Montaguia Charcoti Lamy.}

1906. Monlaguia Charcoli Lamy, Lamellibr. Exp. Charcot, Bull. Mus, Hisl. Nal., t. XII, p. 46.

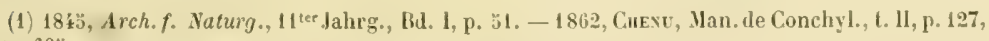
fig. 603 .

(2) 1898, Journ. of Conchol., vol: IX, p. 10f, PI. I, fig. 12. - 1907, Scottish Nation. Antarct. Exped, Moll., Trans. Roy. Soc. Edinburgh, vol. XLVI, p. 149.

(3) 1910, Shells lialkland, Ann. Mag. Nut. Hist., $8^{e}$ série, vol. V, p. 112, Pl. IV, fig. 6. 
1906. M. T'urqueti LAMY, ibid., p. 47.

1906. M. Charcoli LAмy, Expéd. Antarct. Franç.du Dr Charcot, Pélécyp., p. 13, Pl. I, fig. 13-14,

No 123. - Ile Petermann (11 février 1909): I individu (dans l'estomac d'un Poisson).

Nos 142 et 19\%. - Ile Wandel, còte sud ( 16 février 1909) : 1 individus.

No325. - Ile Petermann, nord-est $(16$ octobre 1909): 2 individus.

No 379. - Ile Petermann, cote est $(18$ novembre 1909): I individu.

Genre PSEUDOKELLYA Pelseneer, 1903.

\section{Pseudokellya cardiformis E.-A. Smith.}

1885. Kellya cardiformis E.-A. Surr, Rep. "Challenger ", Lamellibr., p. 202, Pl. XI, fig. 6-6 $b$.

1903. Pseudokellya cardiformis Sm., Pelseneer, Voy. "Belgica ", Moll., p. 48.

No 649. - Port-Lockroy, chenal de Roosen, dragage XV, 70 mètres (26 novembre 1909): 1 individu.

Ce spécimen, par son contour comme par sa sculpture consistant en cotes rayonnantes très fines et serrées, me paraît identique à cette espèce de Kerguelen.

Genre KELLYA Turton, 1822.

Kellya suborbicularis Montagu.

1803. Mya suborbicularis Montagu, Test. Britann., p. 39 et p. 564, Suppl., PI. XXVI, fig. 6 .

1848. Kellia suborbicularis Mtg., Tunton, Conch. Dithyra Britann., p. 57, Pl. XI, fig. 5-6. 1885. K. suborbicularis Mtg., E.-A. Sмiтн, Rep. "Challenger", Lamellibr, p. 201.

No 649. - Port-Lockroy, chenal de Roosen, dragage XV, 70 mètres (26 novembre 1909): 1 individu.

Sauf un moindre développement des dents de la charnière, cette coquille

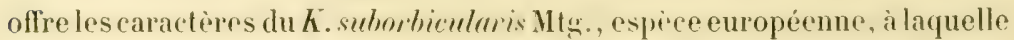
M. E.-A. Smith a identifié complètement une forme de Kerguelen.

Kellya simulans E.-A. Smith.

1907. Kellia simulans E.-A. Sмгтн, Nation. Anlarcl. Exped. "Discovery ", Lamellibr., p. 2, Pl. III, fig. 1 . 
No 649. - Port-Lockroy, chenal de Roosen, dragage XV, 70 mètres (26 novembre 1909): 1 individu.

Ce petit spécimen, qui a un diamètre antéro-postéricur de 3 millimètres et un diamètre umbono-ventral de $2^{\text {ш๘ }}, \ddot{3}$, ne me semble pas pouvoir être distinguée spécifiquement du K. simulans: cependant ses sommets sont un peu moins saillants que ne l'indique la figure de II. E.-A. Smith.

\section{Genre CARDITA Bruguière, 1792. \\ Cardita astartoldes von Martens.}

1878. Cardila aslarloides v. Martens, Silzungsber. Ges. Naturf. Fr. Berlin, p. 25.

1885. C. aslarloides IIrts., E.-A. Sмiти, Rep. "Challenger", Lamellibr., p. 212, Pl. XV,

fig. $2-2 \mathrm{c}$.

1902. G. astartoides Mrts., E.-A. Sмiтн, Voy. "Southern Cross », Moll., p. 211.

1906. G. aslarloides Mrts., LAmy, Expéd. Anlarct. Franç. du Dr Charcot, Pélécyp., p. 14.

1907. G. aslarloides Mrts., E.-A. Sмiтн, Nalion. Antarct. Exped. "Discovery", Lamellibr., p. ?.

No 34. - Près de la Terre Alexandre-I ${ }^{\mathrm{e}}$, dragage VII, 250 mètres (16 janvier 1909): 1 individu.

No 626. - Ile Petermann, côte nord-est, chenal de Lemaire, dragage XIII $b, 30$ à 15 mètres $(17$ novembre 1909): 1 individu.

No647. - lle Petermann: une valve isolée.

\section{Genre ANATINA Lamarek, 1809.}

Anatina elliptica King et Broderip.

1831. Analina elliplica Krng et Broderip, Zool. Journ., vol. V, p. 335.

1834. A. prismalica Sowerby, Proc. Zool. Soc. London, p. 87.

1860. A. elliplica $\mathrm{Kg}$. et Br., ReEve, Conch. Icon., vol. XIV, Anatina, Pl. II, fig. 14.

1885. A. elliplica Kg. et Br., E.-A. Smitu., Rep. "Challenger ", Lamellibr., p. 76.

1902. A. elliplica Kg. et Br., E.-A. Smirn, Voy. "Southern Gross", Moll., p. 210, Pl. XXV, fig. 9-10.

1906. A. elliplica Kg. et Br., LAмy, Expéd. Anlarct. Franç. du Dr Charcot, Pélécyp., p. 14.

1907. A. elliplica Kg. et Br., E.-A. Sмгтн, Nalion. Anlarct. Exped. "Discovery", Lamellibr., p. 1, PI. III, fig. 3.

1907. A. elliplica Kg. et Br., Melvill et Standen, Scollish Nation. Antarct. Exped., Moll., Trans. Roy. Soc. Edinburgh, vol. XLVI, p. 151.

No 123. - Ile Petermann (11 février 1909): 2 jeunes individus (dans l'estomac d'un Poisson). 
No 327. - Grève de Yankee Ilarbour, île Déception, Shetlands du Sud (décembre 1909): 1 individu en débris.

\section{Genre TIIRACIA Blainville, 1824.}

Thracia meridionalis E.-A. Smith.

1885. Thracia meridionalis E.-A. Smitr, Rep. "Challenger ", Lamellibr., p. 68, Pl.VI, fig. $4-4 \cdot b$.

1906. T. meridionalis Sm., LaMy, Expéd. Anlarcl. Franç. du Dr Gharcot, Pélécyp., p. 15. 1907. T. meridionalis E.-A. Smith, Nalion. Antarct. Exped. "Discovery », Lamellibr., p. 1.

No 24:- - Ile Petermann: 1 individu.

No 617. - Port-Lockroy, chenal de Roosen, dragage XV, 70 mètres (26 novembre 1909): 1 individu.

No 661.- Ile Déception, milieu de Port-Foster, dragage XVI, 1300 mètres (9) décembre 1909): 3 individus.

Genre RADULA Rumphius, 1710.

Radula (Limatula) pygmea Philippi.

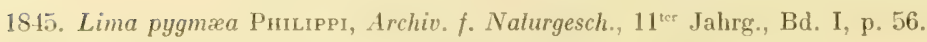

1863. Limalula falklandica A. Adaus, Proc. Zool. Soc. London, p. 509.

1872. Lima falklandica A. Ad., Reeve, Conch. Icon., vol. XVIII, Lima, Pl. V, fig. 24. 1879. Radula (Limalula) pygmæa Phil., E.-A. Smitr, Moll. Kerguelen, Phil. Trans.

Roy. Soc. London, vol. CLXVIII, p. 191, PI. IX, fig. 16.

1885. Lima (Limalula) pygmæa Phil., E.-A. Smitr, Rep. "Challenger ", Lamellibr., p. 292.

1906. Radula (Limalula) pygmæa Pliil, LAмy, Expéd. Antarct. Franç. du Dr Charcot, Pélécyp., p. 15.

1907. Lima (Limalula) pygmrea Phil, Melvill et Standen, Scollish Nalion. Antarcl.

Exped., Moll., Trans. Roy. Soc. Edinburgh, vol. XLVI, p. 148.

No 7. - Port-Lockroy, chenal de Roosen (23 décembre 1908): 30 individus.

No 330. - Ile Petermann (octobre 1909): 1 individu.

No 379. - Ile Petermann, còte est (17-18 novembre 1909): 2 individus.

No 432. - lle Petermann : 20 individus. 


\section{Genre PECTEN Belon, $13: 33$. \\ Pecten Colbecki E.-A. Smith.}

1902. Peclen Colbechi E.-A. Surti, Voy. "Southern Cross 》, Moll., p. 212, Pl. XXV. fig. 11 .

1903. P. Racovilzai Pelseneer, Voy. "Belgica ", Moll., p. 27, Pl. VIII, fig. 101-102. 1906. P. Racovitzai Pels., LAmy, Expéd. Anlarcl. Franç. du $D^{\mathrm{r}}$ Charcot, Pélécyp., p. 16, Pl. I, fig. 19, :01, : 1 .

1907. P. Colbecki E.-A. Smin, Nalion. Antarct. Exped. "Discovery ", Lamellibr., p. 6, Pl. III, fig. 9-9 $a$.

1907. P. Colbecki Sm., Melvill et Standen, Scollish Nalion. Anlarct. Exped., Moll., Trans. Roy. Soc. Edinburgh, vol. XLVI, p. 146.

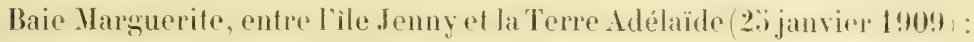
1 individu.

Il ne parait pas y avoir de différence entre le Pecten, de couleur rougeâtre, orné de còtes rayonnantes assez faibles et de fines stries concentriques légèrement saillantes, décrit par M. L. -A. Smith, en 1902, sous le nom de P. Colbeclí et celui appelé par 11 . Pelseneer, en 1903, P. Racovitän.

\section{Genre AMUSS/UII Klein, 1733.}

$$
\text { Amussium clathratum von Martens. }
$$

1881. Pecten clathralus v. Martens, Silzungsb. Ges. Nalurt. Fr. Berlin, p. 79.

1885. P. clalhratus Mrts., E.-A. Suith, Rep. "Challenger ", Lamellibr., p. 305, PI. XXII, fig. 4-4 $a$.

No 286. - Près de la Terre Alexandre-I ${ }^{\text {er }}$, dragage $\mathrm{X}$, 297 mètres (22 janvier 1909): 2 individus.

Ces spécimens me semblent correspondre très exactement à la deseription et aux figures données par M. E.-A. Smith pour cette espèce de Kerguelen: I'Amussium octodecim-liratum Melvill et Standen (1), de l'Antaretique, est une forme voisine qui se distingue par sa taille plus grande et sa coquille plus transverse.

\section{Genre NODIOLARCA Gray, 1847. \\ Modiolarca trapezina Lamarck.}

1819. Modiola trapesina Lamarck, Anim. s. vert., t. VI, $1^{\text {re }}$ p., p. 114.

(1) 1907, Scottish Nation. Antarct. Exped., Moll., Trans. Roy. Soc. Edinburgh, vol. XLV'I, p. 147, Pl. I, fig. 17-17a. 
1841. M. trapesina Lk., Delessent, Rec. Coq. Lamarck, Pl. XIII, fig. 7.

1854. Phaseolicama trapezina Lk., Hupé, in GAY, Hist. Chile, Zool, VIII, p. 323, Pl. VIII, fig. 9.

1852-56. Gaimardia Irapesina Lk., Gould, U. S. Exp. Wilkes, Moll., p. 459, Pl. XLI, fig. $568 a-f$.

1879. Modiolarca trapezina Lk., E.-A. Sмrтн, Moll. Kerguelen, Phil. Trans, Roy. Soc. London, vol. CLXVIII, p. 190.

1885. M. Irapezina Lk., E.-A. Sмітн, Rep. "Challenger ", Lamellibr., p. 279.

1886. M. trapezina Lk., v. Martens et Pfeffer, Moll. Süd-Georgien, Jahrb. Hamburg. Wiss, Anst., III, p. 127, Pl. IV, fig. 13.

1889. M. trapezina Gray, de Rochebrune et Mabille, Miss. Scient, cap Horn., Moll. p. 120 .

1898. M. Trapezina Lk., Melvill et Standen, Shells Falklands, Journ. of Conchol. vol. IX, p. 104.

1901. M. Irapezina Lk., Melvill et Standen, ibid., vol. X, p. 47.

1902. M. trapezina Lk., Stempeld, Musch. Samml. Plate, Fauna Chilensis, Zool. Jahrb., Suppl. V, p. 227.

1903. M. Irapezina Lk., Pelseneer, Voy. "Belgica ", Moll., p. 11 et p. 44, Pl. VIII, fig. 106.

1910. M. trapezina Lk., Lamy, Moll. Kerguelen, Bull. Mus, Hist. Nal., t. XVI, p. 204. 1911. M. Irapezina Lk., Lamy, Moll. Géorgie du Sud, Bull. Mus. Hist. Nal., t. XVII, p. 27.

No 29\%. - Près de la Terre Alexandre. ${ }^{\mathrm{er}}$, dragage $\mathbf{X}$, 297 mètres (22 janvier 1909$)$ : 2 individus.

De Rochebrune et Mabille (1) ont décrit sept espèces de Modiolarca, de la baie Orange: crassa, Le Camellieri, Lephayi, Sacatieri, fuegiensis, Sauvineti, IIalmi, qui sont, commele dit avec raison M. W. Stempell, tout au plus des variétés du H. trapezina Lk.

D'autre part, contrairement à M. E.-A. Smith, ils regardaient le Phaseolicama magellanica Rousseau (2) non seulement comme n'étant pas synonyme du Vorlohlore trapezina, mais comme appartenant ì un genre différent : cette séparation est admise par $\mathbf{I I}$. Stempell.

\section{Genre PIILOBRYA Carpenter, 1872.}

Philobrya wandel.ensis Lamy.

1906. Philobrya wandelensis Lamy, Lamellibr. Expéd. Charcot, Bull. Mus. Hist. Nal., t. NII, p. 50.

1906. P. ualandelensis Lany, Expéd. Anlarct. Franç. du Dr Charcol, Pélécyp., p. 17, PI. I, fig. $15-16$.

(1) 1889, Miss. Scient. Cap Horn, Moll, p. 120-123, Pl. VII, fig. 1-7.

(2) 185i, Voy. Pôle Sud, Zool., V, Moll., p. 116, PI. XXVI, fig. 2 a-d. 
1907. P. wandelensis Lamy, Melvilu et Standen, Scollish Nalion, Anlarcl. Exped., Moll., Trans. Roy. Soc. Edinburgh, vol. XLVI, p. 146.

No 312 . - Ile Petermann, anse nord (10 octobre 1909) : 1 individu. Nos 364 et 432 . L Ile Petermann : 3 individus.

Cette espèce, que j'ai décrite comme nouvelle parmi les Hollusques rapportés par la première expédition du $D^{c}$ Charcot, a été retrouvée aux Orcades du Sud par l'expédition antaretique écossaise.

Philobrya sublevis Pelseneer.

1903. Philobrya sublavis Pelseneer, Voy. "Belgica ", Iloll., p. 25, Pl. VII, fig. 93-94. 1906. P. sublævis Pels., Lany, Lamellibr. Expéd. Charcot, Bull. Mus. Hist. Nat., t. XII, p. 51 .

1906. P. sublixvis Pels., Lııy, Expéd. Antarct. Franç. du Dr Charcol, Pélécyp., p. 18,

Pl. I, fig. 17-18.

No 7. - Port-Lockroy, chenal de Roosen (23 décembre 1908) : 1 individı.

No 286. - Près de la Terre Alexandre-I ${ }^{\text {cr }}$, dragage $\mathrm{X}, 297$ mètres (22 janvier 1909$)$ : \& individus.

No T/4. - Anse ouest de la baie de l'Amirauté, ile du Roi-George, Shetlands du Sud, dragage XVIII, 7j mètres (27 décembre 1909): 9 individus.

Parmi les Philobrya signalés de l'Antaretique, le $P$. meridionalis

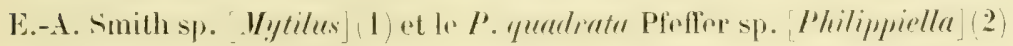
se distinguent de ce $P$. sublevis par leurs sommets plus saillants et leur sculpture bien moins marquéc; le P. limoides E.-A. Smith (3), qui a, lui, un aspect extérieur extrèmement voisin, se diflérencierait surtout par l'absence de bande crénelée à la charnière.

Genre LIMOPSIS Sassi, 1827.

Limopsis mitella Mabille et de Rochebrune.

(Pl. I, fig. 18-19-20.)

1889. Limopsis hirtella Mabille et de Roghebrune, Miss. Scient. Cap. Horn, Moll; p. 115 .

(1) 1885, Rep. "Challenger ", Lamellibr., p. 273, PI. XVI, fig. 3-3 a.- 1907, MeLriel et Stavdes, Scottish Natinn. Antarct. Exped., Moll., Trans. Roy. Soc. Edinburgh, vol. XLVI, P. 145.

(2) 1886, v. Mantevs et Pfeffer, Holl. Sud-lieorgien, Jahrb. Hamburg. Wiss. Anst., III, p. 119, Pl. IV, fig. 6a-b. - 1907, Melvill et Standen, loc. cit., p. 143.

(3) 1907, Nation. Antarct. Exped. "Discovery ", Lamellibr., p. \&, P1. III, tig. 2-2 b.

2* Expédition Charcot. - LAwY. - Gastropodes prosobranches, Scaphopode et Pélécypodes. 
1906. L. hirlella Mab, et Rochbr., LAmy, Expéd. Anlarcl. Franç. du Dr Charcol, Pélécyp.; p. 18.

No6.47. - Ile Petermann : 1 individu.

Le $L$. hirtellı, dont est voisin le $L$. longipilosa Pelseneer (1), est représenté dans les collections du Muséum de Paris par trois exemplaires, de la baie Orange, étudiés par Mabille et de Rochebrune; cette es rèce n'ayant pas encore été figurée, deux de ces types sont dessinés dans la planche qui aceompagne le présent mémoire: le plus grand diam. ant.-post. : $11^{\mathrm{m}^{m}}$; diam. umbono-ventr. : $14^{\mathrm{m}} .6$; épaiss. : $9^{\mathrm{mm}}$ possède une coquille arrondie légèrement trigone (II. I, fig. 18-19); mais le plus petit (diam. ant.-post. : $11^{\mathrm{mm}}$; diam. umb.-ventr. : $11^{\mathrm{mm}}$; épaiss. : $6^{\mathrm{mm}}$ ) a un contour bien plus nettement orbiculaire ( P. I, fig. 20). C'est plutôt de cette dernière forme que se rapprochait un spécimen (diam. ant.post. : $14^{\mathrm{mm}}$; diam. umb.-ventr.: $13^{\mathrm{mm}}$; épaiss. $8^{\mathrm{mm}}$, ij) recueilli à l’ile Anvers par la première expédition du $D^{r}$ Charcol, et c'est également le cas de l'échantillon qui a été recueilli à l'île P'etermann par M. le $\mathrm{U}^{\mathrm{r}}$ Liouville, mais qui oflre de plus grandes dimensions (diam. ant.-post. : $18^{\mathrm{mm}},: ;$; diam. umb.ventr. : $18^{\mathrm{mm}}, \ddot{3}$; épaiss. : $\left.11^{\mathrm{mm}},: 3\right)$.

Limorsis Joussenumer Mabille et de Rochebrune.

1889. Felicia Jousseaumi Mabille et de Rochebrune, Miss. Scient. Cap Horn, Moll., p. 116, Pl. VII, fig. 9.

1907. Limopsis grandis E.-A. Suntr, Nalion. Anlarcl. Exped. "Discovery ", Lamellibr., p. 5, PI. III, fig. 7-7 b.

No 29:3. - Pres de la Terre Alexandre-I ${ }^{\text {er }}$, dragage $\mathrm{X}, 297$ mètres, (22 janvier 1909) : 1 valve isolée.

Dans le dragage $\mathbf{X}$, eflectué près de la Terre Alexandre-I ${ }^{\mathrm{er}}$, une grande valve de Limopsis dian. ant.-post. : $36^{\mathrm{mm}}$; diam. umb.-ventr. : $33^{\mathrm{mm}}$; épaiss. $\left.: 3^{\mathrm{m}}, \ddot{j}\right)$ a été recueillie : par tous ses caractères, contour obliquement ovale, forme tris aplatie, bord dorsal rectiligne, impression du muscle adducteur antérieur petite, impression du muscle postérieur allongée et limitée au bord interne par un bourrelet, elle se montre enticrement semblable au $L$. grandis $\mathbf{E}$ - $-\mathbf{A}$. Smith, forne voisine du L. leverusculı Pelseneer (2).

1) 1903, Voy. "Belyzica », Moll., p.23, pl. l'll, fig. 89-90.

(2) 1503, Voy. "Belgica ", Holl., p. 2i, Pl. VII, fig. 91-92. 
Mais, d'autre part, la comparaison de cette valve avec les types du Felicin Joussenmei Nab. et Rochbr., conservés au Muséum de Paris, me porte à admettre qu'elle est identique à cette espèce, les diflérences tenant simplement à ceque ces types sont des coquilles à un stade un peu plus jeune.

Or, quant à la valeur de ce genre Felicia, Félix Bernard (I) a déjà recomm que le $l i$. Joussenumei, lorsqu'il a encore d'assez faibles dimensions (12 millimètres), est un véritable Limopsis : il n'y a, par suite, qu'à faire rentrer cette espece dans le genre Limopsis.

Je erois done devoir adopter pour la forme en question le nom de Limopsis Joussenumei, dont L. grandis serait synonyme.

\section{Genre ADAC VAKA Pelseneer, 1903.}

Adacnarca nitens Pelseneer.

1903. Adacnarca nilens Pelseneer, Voy. "Belgica ", Moll., p. 24, PI. VII, fig. 83.

1906. A. nilens Pels., Lamy, Expéd. Anlarcl. Franç. du Dr Charcol., Pélécyp., p. 19.

1907. A. nilens Pels., E.-A. Smiru, Nation. Antarcl. Exped. "Discovery ", Lamellibi., p. j, I'. II, fig. $6 a-c$.

Yo 36. - Prés de la Terre Alexandre-I ${ }^{\mathrm{er}}$, dragage VII, 2000 métres $(16$ janvier 1909): 1 individu.

Xo 286. - Pres de la Terre Alexandre-1er, dragage $\mathrm{X}, 297$ metres (22.2 janvier 1909): 1 individu.

\section{Genre IRCA Limné, 17538.}

\section{Arca (Bathyarca) sinuata Pelseneer.}

1903. Balhyarca sinuala Pelseneer, Voy. "Belgica ", Moll., p. 23, PI. VI, fig. 81-82.

No 828. - Par $70 \circ 10^{\prime}$ latitude sud et $800^{\circ} ; 0^{\prime}$ longitude ouest, en bordure de la banquise, dragage XX, 160 metres 12 janvier 1910): 2 individus.

Le plus grand de ces deux spécimens correspond exactement aux dimensions données par. M. Pelseneer (diam. ant.-post. : $11^{\text {mm }}$; diam. umb.-ventr. : $7^{\mathrm{mm}}$; épaiss. : $\left.6^{\mathrm{mm}}\right)(\boldsymbol{2})$.

(1) 1898, Kech. Corf. Lamellibr, Ann. Se, nat., 8e série, Zool., t. VIll, p. 107.

(2) Comme le montre la figure donnée par 11 . Pelseneer, qui est grossie trois fois, il y a erreur d'im ression dans sa description, qui indique 17 millimètres de hauteur, au lieu de 7 millimètres. 
Arca (Batmirca) Goundoni Lamy.

(Pl. I, fig. 21-2?.)

1910. Arca (Balhyarca) Gourdoni Lisy, Pélécyp. Mliss. Charcot., Bull. Mus. Hisl. Nal., t. XVI, p. 393.

Testa paru, altior quam latu, subquadrato-ovata, obliqua, gibbosu, temuis, avphivalvis, valde imequilateralis, antice pro bysso leviter hians, striis incrementi concentricis ornata, allida, epidermide griseo-luteat induta. Latus anticum breve; latus posticum paululum expansum. Margo dorsualis rectus, utrinque subangulatus; margo ventratis arcuatus. Umbones prominentes, antrorsum inclinati. Cardo subarcuatus, utrinque dentibus paucis munitus. Valiarum pagina interna radiatim substriata: strix lactanex extus conspiciuntur; margines crenati; impressiones musculares inconspicuie.

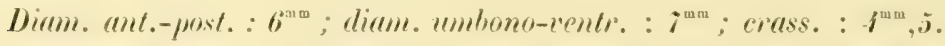

Coquille petite, plus haute que large, de forme ovale presque quadrangulaire, oblique, rentlée, mince, équivalve, très inéquilatérale, légèrement bâillante en avant pour le passage du byssus, ornée de stries d'accroissement concentriques, de couleur blanchâtre sous un épiderme gris jaunâtre. Còté antérieur court; côté postérieur un peu dilaté. Bord dorsal rectiligne, subanguleux à ses extrémités; bord ventral arqué. Sommets saillants, inclinés en avant. Charnière légèrement arquée, composée de dents peu nombreuses de chaque côté. Intérieur des valves pourvu de stries rayonnantes superficielles, d'un blane laiteux opaque, qui se voient par transparence sur la face externe de la coquille; bords crénelés; impressions des muscles adducteurs peu visibles.

No 56. - Près de la Terre Alexandre-1 ${ }^{e r}$, dragage VII, $2: 30$ metres ( 16 janvier 1909$)$ : 23 individus.

No 286. - Près de la Terre Alexandre- ${ }^{e r}$, dragage $\mathbf{X}, 297$ mètres (22. janvier 1909$): 14$ individus.

No 36\%. - Ile Petermann: 1 individu.

No 629. - lle Petermann : 1 individu.

Celte coquille, très renflée et plus haute que large, ressemble un peu extérieurement au l'hilobrya wandelensis Lamy; sauf sa taille 
beaucoup plus faible, elle rappelle également par sa forme l'Arę corpulenta E.-A. Smith (1), du Pacifique.

\section{Genre IOLDIA Möller, 1842.}

Yoldia Woodwardi Hanley.

1860. Yoldia Woodivardi Hanley, Proc. Zool. Soc. London, p. 370.

1871. Y. Woodwardi Hanl. ReEve, Conch. Icon., vol. XVIII, Yoldia, Pl. I, fig. 2.

1903. Y. Woodwardi Hanl., Pelseneer, Voy. "Belgica ", Moll., p. 10.

1906. Y. Woodwardi Hanl., Lam, Moll. Orcades du Sud, Bull. Mus. Hist. Nat, t. XII, p. 125 .

1906. Y. Woodwardi Hanl., LaMy, Expéd. Antarct. Franç. du Dr Charcot, Pélécyp., p. 19.

No 7. - Port-Lockroy, chenal de Roosen (23 décembre 1908) : 1 individu.

Nos 123 et 133. - Ile Potermann (11 février 1909) : 3 individus (dans l'estomac d'un Poisson).

No 172. - Ile Petermann (février 1909) : 6 individus (dans l'estomac d'un Poisson).

No 245 . - Ile Petermann : 12 individus.

No 661. - Ile Déception, milieu de Port-Foster, dragage XVI, 150 mètres (9 décembre 1909) : 4 individus.

\section{Yoldia INEQUiSculPta Lamy.}

(Pl. I, fig. 23.)

1906. Yoldia inæquisculpla LAMr, Moll. Orcades du Sud, Bull. Mus, Hisl. Nal., t. XII, p. 125, fig. 3 .

No 243. - Ile Petermann : 60 individus.

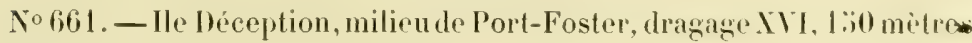
(9 décembre 1909): 2 individus.

- No 714. - Anse ouest de la baie de l'Amirauté, île du hoi-George, Shetlands du Sud, 75 mètres (27 décembre 1909) : 2 individus.

Toutes ces corfuilles, de forme ovale et transverse, de couleur jaune verdàtre, sont ornées de fines stries concentriques qui sont bien marquées sur le còté antérieur assez court et arrondi, ainsi que sur le milieu du test, mais qui ne se prolongent pas sur le côté postérieur allongé et

(1) 1885, Rep. "Challenger ", Lamellibr., p. 263, Pl. XVII, fig. 5-5 6. 
obliquement tronqué; elles peuvent atteindre les dimensions suivantes: diam. ant.-post. : $16^{\mathrm{mm}}$; diam. umb.-rentr. : ( $^{\mathrm{mm}}$; épaiss. : $6^{\text {mm }}$; sauf cette taille plus grande, elles sont identiques ì une espèce recueillie aux Orcades du Sud par II. Valette en 1904 et que j’ai décrite en 1906 sous le nom d'Y. iniequisculple.

\section{Gemre s/LITILA Jeffress, 1879.}

Silicula Rouchi Lamy.

(Pl. 1, fig. 24-25.)

1910. Silicula Rouchi Lavy, Pélécyp. Miss. Charcot, Bull. Mus. Hist. Nat., t. XVJ, p. 394.

Testa parea, oblonga, transeorsime elongata, temuis, fiayilis, compressa, iequivale is, inequilateralis, striis incrementi concentricis mrnata, iridesenes,

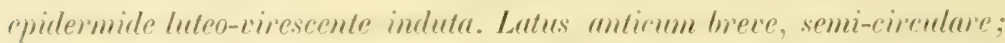
Iatus pasticum productum, sublruncatum. Maryo dorsuatis fere rectus, margo ientralis arcuatus. Cmbones acuti, frominentes. Cardo in medio

- edentulus, utrinque dentibus lamellosis, mrelongis, angustis, transeersalibus, anm margine dorsuati paralletis, antice tribus, postice quatuon lon-

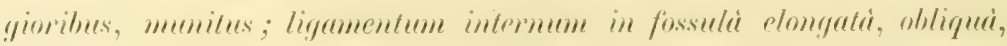
insertum. Talearam pagina interna nitidula; impressiones mustalares

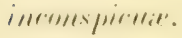

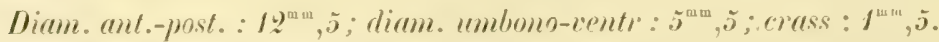

Coquille petite, oblongue, de forme transverse, mince, fragile, comprimée, équivalve, inéquilatérale, pourvue seulement de stries d'aceroissement, irisée sous un épiderme jaune verdàtre. Cốé antérieur court, semi-circulaire; còté postérieur allongé, légèrement tronqué. Bord dorsal presque droil, bord ventral arqué. Sommets aigus, saillants. Charnière sans dents au milieu, pourvue de chaque coté de dents lamelleuses allongées, étroites, transversales, parallèles au bord dorsal, au nombre de trois en avant et de quatre plus longues en arrière; ligament interne s'attachant dans une fossette allongée et oblique. Intérieur des valves légèrement luisant; impressions des muscles adducteurs peu visibles. 
$N^{0}$ :4. - Près de la Terre Alexandre-I ${ }^{\mathrm{Ir}}$, dragage VII, $2: 30$ mètres (16 janvier 1909): 1 individu.

Le seul Mollusque auquel je puisse comparer cette très intéressante coquille, allongée et aplatie, avec sa charnière à longues dents lamelleuses et son ligament interne, est le Silicula fragilis. Jefreys (1), de l'Atlantique (2); c'est pourtant avec un certain doute que je la place dans ce genre Silicula $=$ Phaseolus, qui renferme également deux espèces méditerranéennes•: Silicula ockala Jeflr. et S.tumidula Nonterosato (3).

(1) 18\%9, Moll. "Lightning " a, "Porcupine" Exped., Pruc. Zool. Soc. London, p. 574, Pl. XLY, fig. 6.

() D'après Jelfreys, dans son espèce, le còté le plus développé serait, au contraire, le cûté antécieur.

(3) 1878 , Enum, e Sinon. Conch. Mediterr., Giorn. Soc. Sc. Nat. ed Econ. Palermo, vol. XlII, p. $67 ; 1880$, Conch. zona abissi, Bu!l. Soc. Malac. Ital., vol. W, p. 56. 


\section{EXPLICATION DE LA PLANCHE I}

Fig. 1-2. - Buccinum Charcoti Ed. L. (Shetlands du Sud).............. $\times 4 / 3$

Fig. 3-4. - Buccinum sp. juv. (Shetlands du Sud) ................. $\times 3$

Fig. 5. - Cominella vestita $v$. Mart. (Kerguelen).................. $\times 4 / 3$

Fig. 6. $\quad-\quad-\quad$ var. elongata Ed. L. (Shetlands du Sud) ......... $\times 4 / 3$

Fig. 7. - Sipho Gaini Ed. L. (Shetlands du Sud) ................. $\times 4 / 3$

Fig. 8. $\quad-\quad-\quad$ - opercule ............................. $\times 4$.

Fig. 9. - Cerithium Liouvillei Ed. L. (PortLockroy).................. $\times 7$

Fig. 10-11. - Natica Godfroyi Ed. L. (Shetlands du Sud) .............. $\times 2$

Fig. 12. - Talvatella sp. juv. (Ile Petermann) .................... $\times 20$

Fig. 13. - Valvatella altera sp. juv. (Orcades du Sud) ................ $\times 20$

Fig. 14-15-16. - Scissurella petermannensis Ed. L. (Ile Petermann).......... $\times 20$

Fig. 17. - Axinus Bongraini Ed. L. (Ile Petermann) ................ $\times 5$

Fig. 18-19. - Limopsis hirtella Mab. et Rochbr. (type: Baie Orange)......... $\times 2$

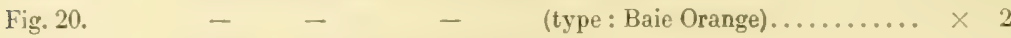

Fig. 21-22. - Arca Gourdoni Ed. L. (Terre Alexandre-Ier)............... $\times 5$

Fig. 23. - Yoldia inæquisculpta Ed. L. (Ile Petermann) ................ $\times 3$

Fig. 24-25. - Silicula Rouchi Ed. L. (Terre Alexandre-Ier) ............. $\times 5$ 

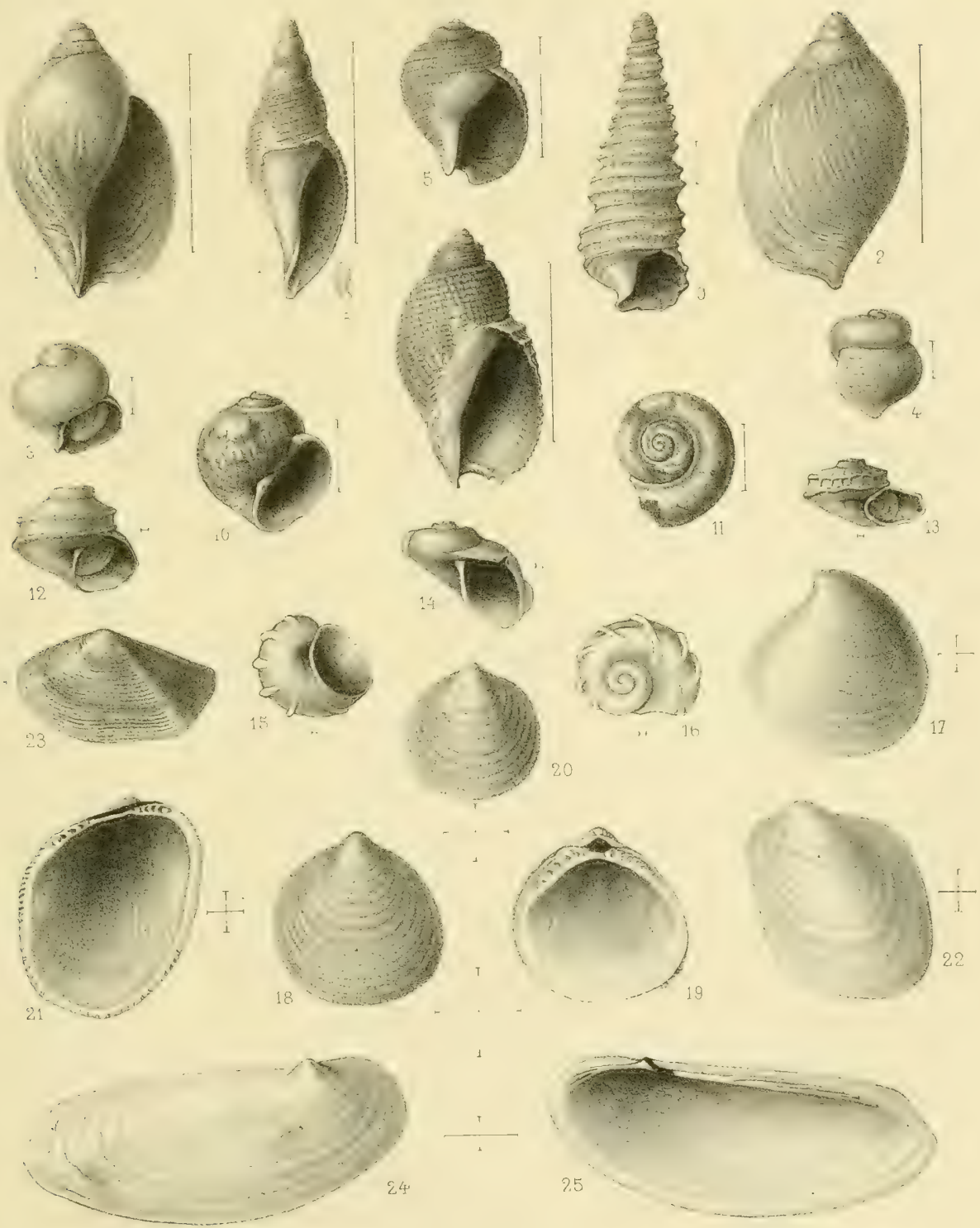



\section{A MPHINEURES \\ Déterminés par le $\mathrm{D}^{\mathrm{r}}$ Joh. THIELE (1)}

Lepidopleurus Kerguelensis (Haddon).

No 330. - Ile Petermann (octobre 1909): 3 individus.

No 346 . - Ile Petermann, nord-est $(30$ octobre 1909$): 6$ individus.

No 379. - Ile Petermann, cote est $(17-18$ novembre 1909$)$ : 亿 individus.

Hemiarthrum setulosum Dall.

No 271. - Ile Petermann, còte nord-est, chenal de Lemaire, dragage XIV $c, 60$ à 40 mètres (18 novembre 1909): 1 individu.

No 35̆. - Baie Mlatha, dragage XI, 380 mètres (I février 1909): 1 individu.

Notochrton Mrandus Thiele.

$\mathrm{X}^{\circ} 110$ et 111. - Au sud de l'île Jenny, dragage IX, 250 mètres (21 janvier 1909): 2 individus.

Callochiton (Icoplax) Bouveti Thiele.

No 58. - Baie Marguerite, dragage VI, $2: 4$ mètres (15 janvier 1909): 2 individus.

No 271. - Ile Petermann, còte nord-est, chenal de Lemaire, dragage $\mathrm{XIV} e, 60$ à 40 mètres (18 novembre 1909): 1 individu.

Plaxiphora setigera (King).

No 866. - Ile Petermann (printemps 1909): I individu.

(1) Voir J. THELE, Die antarktischen und subantarktischen Chitonen (Deutsche Südpolar Expedition 1901-1903, Bd. X, Zool., II, p. s-23, P1. I, Berlin, 1908).

¿o Expédition Charcot. - Thele. - Amphineures. 
Tonicina Zschaui (Pfeffer).

No 271. - Ile Petermann, côtenord-est, chenal de Lemaire, dragage XIV $c, 60$ ì 60 mètres (18 novembre 1909): 2 individus.

Nos 296 et 297 . - Ile Petermann (2 et 3 octobre 1909): 2 individus.

No 306. - Ile Petermann, anse nord (10 octobre 1909): 1 individu.

No 349. - He Petermann (30 octobre 1909) : 2 individus.

$$
\text { Ginton subfuscus Sowerby: }
$$

No 761. - Baie Tuesday (Magellan) : 1 individu. 
Corbeil. - Imprimerie Grété. 


cas का एर

$\left.\right|_{0 \text { क्षा की }}$

in $\leq<0$

into

5

14955 - Conerit: Imoprimerle Caśr

14955 - Conesti: Imprimerle Castre
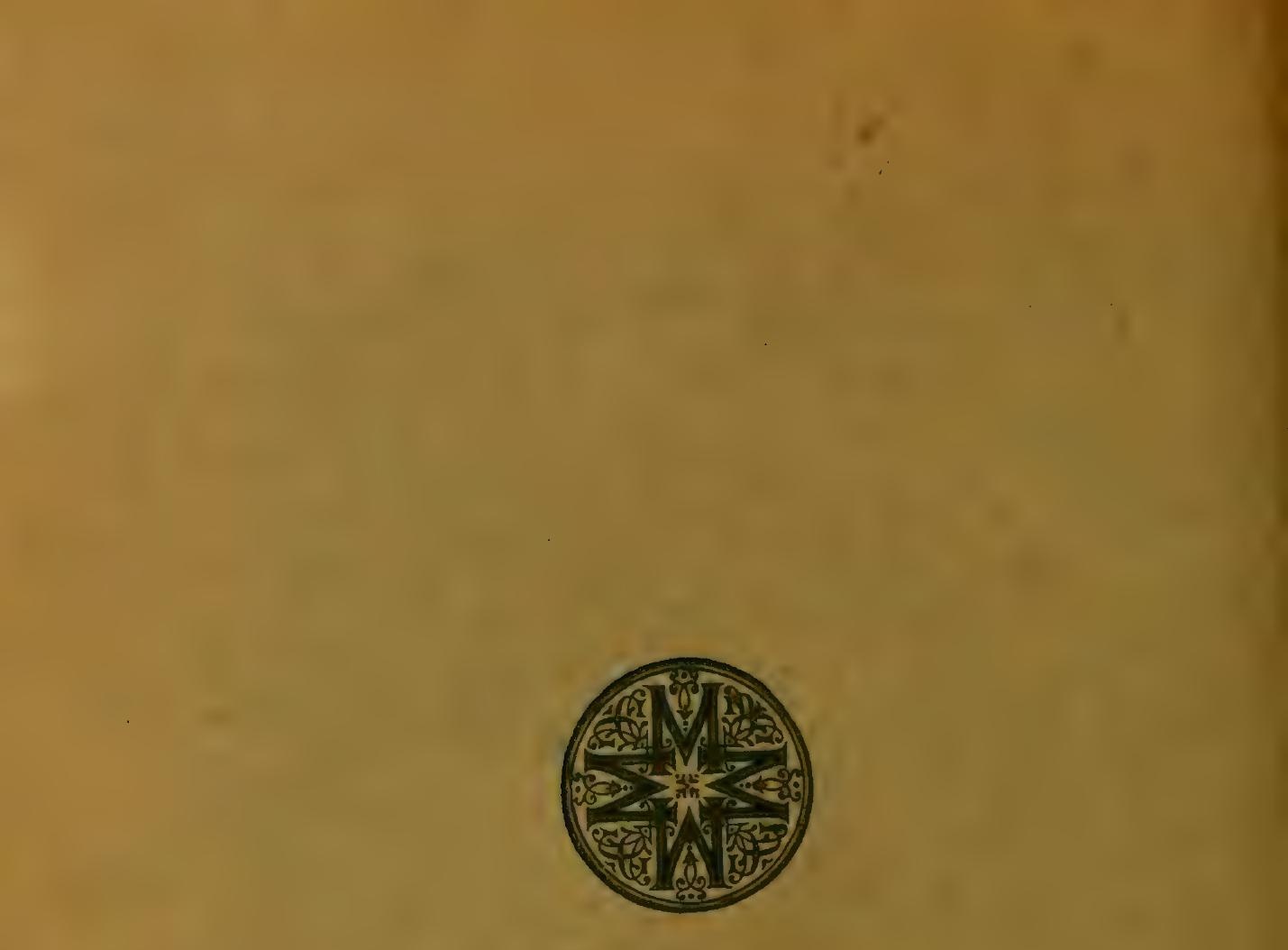

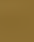























